\title{
Measurement of $\eta_{c}(1 S), \eta_{c}(2 S)$, and nonresonant $\eta^{\prime} \pi^{+} \pi^{-}$ production via two-photon collisions
}

\author{
Q. N. Xu, ${ }^{20, *}$ I. Adachi, ${ }^{12,9}$ H. Aihara,${ }^{74}$ S. Al Said, ${ }^{69,31}$ D. M. Asner ${ }^{58}$ H. Atmacan, ${ }^{65}$ V. Aulchenko,, 56
} T. Aushev, ${ }^{47}$ R. Ayad, ${ }^{69}$ V. Babu, ${ }^{70}$ I. Badhrees,${ }^{69,30}$ A. M. Bakich, ${ }^{68}$ V. Bansal,${ }^{58}$ E. Barberio, ${ }^{43}$ P. Behera, ${ }^{18}$ M. Berger, ${ }^{66}$ V. Bhardwaj, ${ }^{15}$ B. Bhuyan, ${ }^{17}$ J. Biswal, ${ }^{26}$ A. Bondar, ${ }^{3,56}$ A. Bozek, ${ }^{53}$ M. Bračko, ${ }^{41,26}$ D. Červenkov, ${ }^{4}$ A. Chen, ${ }^{50}$ B. G. Cheon, ${ }^{10}$ K. Chilikin, ${ }^{36,46}$ K. Cho, ${ }^{32}$ S.-K. Choi ${ }^{82}$ Y. Choi, ${ }^{67}$ D. Cinabro, ${ }^{79}$ S. Cunliffe, ${ }^{7}$ T. Czank, ${ }^{72}$ N. Dash, ${ }^{16}$ S. Di Carlo ${ }^{79}$ Z. Doležal, ${ }^{4}$ Z. Drásal ${ }^{4}$ S. Eidelman, ${ }^{3,56,36}$ D. Epifanov, ${ }^{3,56}$ J. E. Fast, ${ }^{58}$ T. Ferber, ${ }^{7}$ B. G. Fulsom, ${ }^{58}$ R. Garg, ${ }^{59}$ V. Gaur, ${ }^{78}$ N. Gabyshev, ${ }^{3,56}$ A. Garmash, ${ }^{3,56}$ M. Gelb,${ }^{28}$ P. Goldenzweig, ${ }^{28}$ Y. Guan,,${ }^{19,12}$ E. Guido ${ }^{24}$ J. Haba, ${ }^{12,9}$ K. Hayasaka ${ }^{55}$ H. Hayashii, ${ }^{49}$ M. T. Hedges, ${ }^{11}$ W.-S. Hou, ${ }^{52}$ K. Inami ${ }^{48}$ G. Inguglia, ${ }^{7}$ A. Ishikawa, ${ }^{72}$ R. Itoh, ${ }^{12,9}$ M. Iwasaki, ${ }^{57}$ Y. Iwasaki, ${ }^{12}$ I. Jaegle, ${ }^{8}$ H. B. Jeon, ${ }^{34}$ Y. Jin,${ }^{74}$ K. K. Joo, ${ }^{5}$ T. Julius, ${ }^{43}$ A. B. Kaliyar, ${ }^{18}$ K. H. Kang,${ }^{34}$ G. Karyan, ${ }^{7}$ T. Kawasaki, ${ }^{55}$ H. Kichimi, ${ }^{12}$ C. Kiesling, ${ }^{42}$ D. Y. Kim, ${ }^{64}$ H. J. Kim, ${ }^{34}$ J. B. Kim, ${ }^{33}$ P. Kodyš, ${ }^{4}$ S. Korpar, ${ }^{41,26}$ D. Kotchetkov, ${ }^{11}$ P. Križan, ${ }^{37,26}$ R. Kroeger, ${ }^{44}$ P. Krokovny, ${ }^{3,56}$ R. Kulasiri, ${ }^{29}$ Y.-J. Kwon ${ }^{81}$ I. S. Lee, ${ }^{10}$ S. C. Lee,${ }^{34}$ L. K. Li,${ }^{20}$ Y. Li ${ }^{78}$ L. Li Gioi, ${ }^{42}$ J. Libby, ${ }^{18}$ D. Liventsev, ${ }^{78,12}$ M. Lubej, ${ }^{26}$ T. Luo, ${ }^{60}$ M. Masuda, ${ }^{73}$ T. Matsuda, ${ }^{45}$ D. Matvienko, ${ }^{3,56,36}$ M. Merola, ${ }^{23}$ K. Miyabayashi, ${ }^{49}$ H. Miyata, ${ }^{55}$ R. Mizuk, ${ }^{36,46,47}$ H. K. Moon, ${ }^{33}$ T. Mori, ${ }^{48}$ R. Mussa, ${ }^{24}$ T. Nanut, ${ }^{26}$ K. J. Nath, ${ }^{17}$ Z. Natkaniec ${ }^{53}$ M. Nayak, ${ }^{79,12}$ N. K. Nisar, ${ }^{60}$ S. Nishida, ${ }^{12,9}$ S. Okuno, ${ }^{27}$ H. Ono, ${ }^{54,55}$ Y. Onuki, ${ }^{74}$ P. Pakhlov ${ }^{36,46}$ G. Pakhlova, ${ }^{36,47}$ B. Pal,${ }^{6}$ H. Park ${ }^{34}$ S. Paul ${ }^{71}$ T. K. Pedlar, ${ }^{39}$ R. Pestotnik, ${ }^{26}$ L. E. Piilonen, ${ }^{78}$ V. Popov, ${ }^{47}$ M. Ritter, ${ }^{38}$ A. Rostomyan, ${ }^{7}$ G. Russo, ${ }^{23}$ Y. Sakai, ${ }^{12,9}$ M. Salehi, ${ }^{40,38}$ S. Sandilya, ${ }^{6}$ T. Sanuki, ${ }^{72}$ V. Savinov, ${ }^{60}$ O. Schneider,${ }^{35}$ G. Schnell, ${ }_{75}^{1,14}$ C. Schwanda, ${ }^{21}$ Y. Seino, ${ }^{55}$ K. Senyo, ${ }^{80}$ O. Seon, ${ }^{48}$ M. E. Sevior, ${ }^{43}$ V. Shebalin, ${ }^{3,56}$ C. P. Shen, ${ }^{2}$ T.-A. Shibata, ${ }^{75}$ N. Shimizu, ${ }^{74}$ J.-G. Shiu, ${ }^{52}$ A. Sokolov, ${ }^{22}$ E. Solovieva, ${ }^{36,47}$ M. Starič ${ }^{26}$ J. F. Strube, ${ }^{58}$ T. Sumiyoshi, ${ }^{76}$ M. Takizawa, ${ }^{63,13,61}$ U. Tamponi,${ }^{24,77}$ K. Tanida ${ }^{25}$ F. Tenchini, ${ }^{43}$ M. Uchida ${ }^{75}$ S. Uehara, ${ }^{12,9}$ T. Uglov, ${ }^{36,47}$ Y. Unno, ${ }^{10}$ S. Uno, ${ }^{12,9}$ P. Urquijo, ${ }^{43}$ C. Van Hulse, ${ }^{1}$ G. Varner, ${ }^{11}$ A. Vinokurova, ${ }^{3,56}$ V. Vorobyev,${ }^{3,56,36}$ A. Vossen,${ }^{19}$ B. Wang, ${ }^{6}$ C. H. Wang, ${ }^{51}$ M.-Z. Wang, ${ }^{52}$ P. Wang, ${ }^{20}$ X. L. Wang, ${ }^{58,12}$ M. Watanabe, ${ }^{55}$ Y. Watanabe, ${ }^{27}$ E. Widmann,${ }^{66}$ E. Won, ${ }^{33}$ H. Ye, ${ }^{7}$ C. Z. Yuan, ${ }^{20}$ Y. Yusa, ${ }^{55}$ S. Zakharov, ${ }^{36}$ C. C. Zhang, ${ }^{20}$ Z. P. Zhang, ${ }^{62}$ V. Zhilich, ${ }^{3,56}$ V. Zhukova, ${ }^{36,46}$ V. Zhulanov, ${ }^{3,56}$ and A. Zupanc ${ }^{37,26}$

(Belle Collaboration)

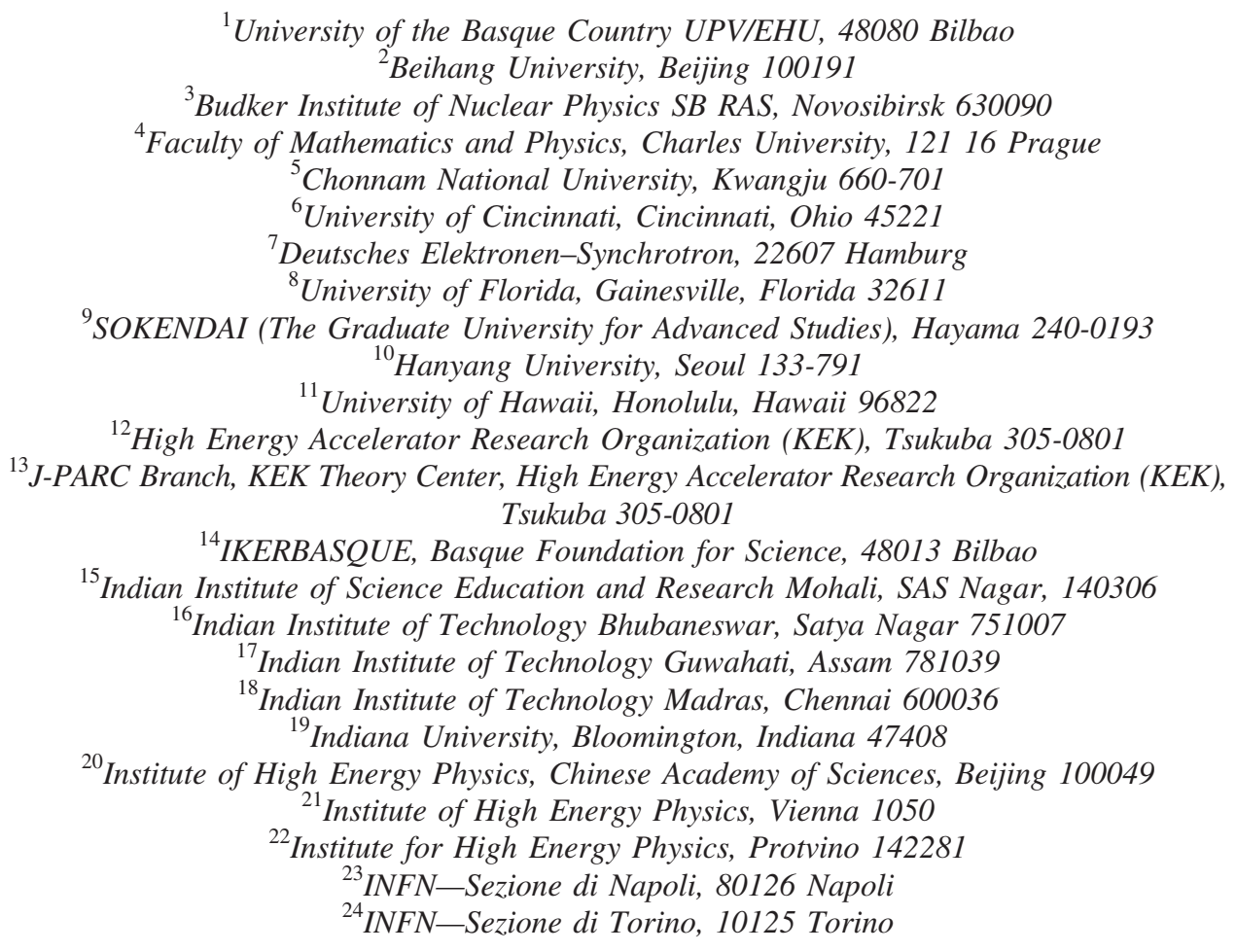

*Also at University of Chinese Academy of Sciences. 
${ }^{25}$ Advanced Science Research Center, Japan Atomic Energy Agency, Naka 319-1195

${ }^{26}$ J. Stefan Institute, 1000 Ljubljana

${ }^{27}$ Kanagawa University, Yokohama 221-8686

${ }^{28}$ Institut für Experimentelle Kernphysik, Karlsruher Institut für Technologie, 76131 Karlsruhe

${ }^{29}$ Kennesaw State University, Kennesaw, Georgia 30144

${ }^{30}$ King Abdulaziz City for Science and Technology, Riyadh 11442

${ }^{31}$ Department of Physics, Faculty of Science, King Abdulaziz University, Jeddah 21589

${ }^{32}$ Korea Institute of Science and Technology Information, Daejeon 305-806

${ }^{33}$ Korea University, Seoul 136-713

${ }^{34}$ Kyungpook National University, Daegu 702-701

${ }^{35}$ École Polytechnique Fédérale de Lausanne (EPFL), Lausanne 1015

${ }^{36}$ P. N. Lebedev Physical Institute of the Russian Academy of Sciences, Moscow 119991

${ }^{37}$ Faculty of Mathematics and Physics, University of Ljubljana, 1000 Ljubljana

${ }^{38}$ Ludwig Maximilians University, 80539 Munich

${ }^{39}$ Luther College, Decorah, Iowa 52101

${ }^{40}$ University of Malaya, 50603 Kuala Lumpur

${ }^{41}$ University of Maribor, 2000 Maribor

${ }^{42}$ Max-Planck-Institut für Physik, 80805 München

${ }^{43}$ School of Physics, University of Melbourne, Victoria 3010

${ }^{44}$ University of Mississippi, University, Mississippi 38677

${ }^{45}$ University of Miyazaki, Miyazaki 889-2192

${ }^{46}$ Moscow Physical Engineering Institute, Moscow 115409

${ }^{47}$ Moscow Institute of Physics and Technology, Moscow Region 141700

${ }^{48}$ Graduate School of Science, Nagoya University, Nagoya 464-8602

${ }^{49}$ Nara Women's University, Nara 630-8506

${ }^{50}$ National Central University, Chung-li 32054

${ }^{51}$ National United University, Miao Li 36003

${ }^{52}$ Department of Physics, National Taiwan University, Taipei 10617

${ }^{53} \mathrm{H}$. Niewodniczanski Institute of Nuclear Physics, Krakow 31-342

${ }^{54}$ Nippon Dental University, Niigata 951-8580

${ }^{55}$ Niigata University, Niigata 950-2181

${ }^{56}$ Novosibirsk State University, Novosibirsk 630090

${ }^{57}$ Osaka City University, Osaka 558-8585

${ }^{58}$ Pacific Northwest National Laboratory, Richland, Washington 99352

${ }^{59}$ Panjab University, Chandigarh 160014

${ }^{60}$ University of Pittsburgh, Pittsburgh, Pennsylvania 15260

${ }^{61}$ Theoretical Research Division, Nishina Center, RIKEN, Saitama 351-0198

${ }^{62}$ University of Science and Technology of China, Hefei 230026

${ }^{63}$ Showa Pharmaceutical University, Tokyo 194-8543

${ }^{64}$ Soongsil University, Seoul 156-743

${ }^{65}$ University of South Carolina, Columbia, South Carolina 29208

${ }^{66}$ Stefan Meyer Institute for Subatomic Physics, Vienna 1090

${ }^{67}$ Sungkyunkwan University, Suwon 440-746

${ }^{68}$ School of Physics, University of Sydney, New South Wales 2006

${ }^{69}$ Department of Physics, Faculty of Science, University of Tabuk, Tabuk 71451

${ }^{70}$ Tata Institute of Fundamental Research, Mumbai 400005

${ }^{71}$ Department of Physics, Technische Universität München, 85748 Garching

${ }^{72}$ Department of Physics, Tohoku University, Sendai 980-8578

${ }^{73}$ Earthquake Research Institute, University of Tokyo, Tokyo 113-0032

${ }^{74}$ Department of Physics, University of Tokyo, Tokyo 113-0033

${ }^{75}$ Tokyo Institute of Technology, Tokyo 152-8550

${ }^{76}$ Tokyo Metropolitan University, Tokyo 192-0397

${ }^{77}$ University of Torino, 10124 Torino

${ }^{78}$ Virginia Polytechnic Institute and State University, Blacksburg, Virginia 24061

${ }^{79}$ Wayne State University, Detroit, Michigan 48202

${ }^{80}$ Yamagata University, Yamagata 990-8560

${ }^{81}$ Yonsei University, Seoul 120-749

${ }^{82}$ Gyeongsang National University, Chinju 660-701 
(Received 9 May 2018; published 3 October 2018)

\begin{abstract}
We report the measurement of $\gamma \gamma \rightarrow \eta_{c}(1 S), \eta_{c}(2 S) \rightarrow \eta^{\prime} \pi^{+} \pi^{-}$with $\eta^{\prime}$ decays to $\gamma \rho$ and $\eta \pi^{+} \pi^{-}$ using $941 \mathrm{fb}^{-1}$ of data collected with the Belle detector at the KEKB asymmetric-energy $e^{+} e^{-}$ collider. The $\eta_{c}(1 S)$ mass and width are measured to be $M=[2984.6 \pm 0.7$ (stat) \pm 2.2 (syst) \pm 0.3 (model) $] \mathrm{MeV} / c^{2}$ and $\Gamma=\left[30.8_{-2.2}^{+2.3}\right.$ (stat) \pm 2.5 (syst) \pm 1.4 (model) $] \mathrm{MeV}$, respectively. First observation of $\eta_{c}(2 S) \rightarrow \eta^{\prime} \pi^{+} \pi^{-}$with a significance of $5.5 \sigma$ including systematic error is obtained, and the $\eta_{c}(2 S)$ mass is measured to be $M=[3635.1 \pm 3.7$ (stat) \pm 2.9 (syst) \pm 0.4 (model) $] \mathrm{MeV} / c^{2}$. The products of the two-photon decay width and branching fraction $(\mathcal{B})$ of decays to $\eta^{\prime} \pi^{+} \pi^{-}$are determined to be $\Gamma_{\gamma \gamma} \Gamma_{\gamma \gamma} \mathcal{B}=[65.4 \pm 2.6$ (stat) \pm 7.8 (syst) $] \mathrm{eV}$ for $\eta_{c}(1 S)$ and $\left[5.6_{-1.1}^{+1.2}\right.$ (stat) \pm 1.1 (syst) $] \mathrm{eV}$ for $\eta_{c}(2 S)$. The cross sections for $\gamma \gamma \rightarrow \eta^{\prime} \pi^{+} \pi^{-}$and $\eta^{\prime} f_{2}(1270)$ are measured for the first time.
\end{abstract}

DOI: 10.1103/PhysRevD.98.072001

\section{INTRODUCTION}

The charmonium states $\eta_{c}(1 S)$ and $\eta_{c}(2 S)$ play an important role in tests of quantum chromodynamics (QCD) [1]. Precise measurement of their two-photon decay widths may provide sensitive tests for QCD models [2]. The lowest heavy-quarkonium state $\eta_{c}(1 S)$, together with the $J / \psi, \eta_{b}(1 S)$, and $\Upsilon(1 S)$, serve as benchmarks for the finetuning of input parameters for QCD calculations [3]. The $\eta_{c}(1 S)$ and $\eta_{c}(2 S)$ resonance parameters were measured in $\psi(2 S)$ radiative decay by BESIII and in $B$ decay and twophoton production by BABAR, Belle and CLEO [4-9]. CLEO made the first measurement of the $\eta_{c}(2 S)$ two-photon decay width $\Gamma_{\gamma \gamma}$ via $K_{S}^{0} K^{+} \pi^{-}$but observed no signal for the $\eta_{c}(2 S) \rightarrow \eta^{\prime} \pi^{+} \pi^{-}$decay [9]. They measured the ratio of the product of $\Gamma_{\gamma \gamma}$ and $\mathcal{B}\left(K_{S}^{0} K^{+} \pi^{-}\right)$for $\eta_{c}(2 S)$ to that for $\eta_{c}(1 S)$, as well as $\Gamma_{\gamma \gamma}$ for $\eta_{c}(1 S)$. Assuming equal $\mathcal{B}$ for the $\eta_{c}(1 S)$ and $\eta_{c}(2 S)$ decays, the two-photon width $\Gamma_{\gamma \gamma}$ for $\eta_{c}(2 S)$ is estimated to be $(1.3 \pm 0.6) \mathrm{keV}$. On the other hand, the assumption of equal $\mathcal{B}$ for $\eta_{c}(1 S)$ and $\eta_{c}(2 S)$ seems implausible since the value of $\mathcal{B}\left(\eta_{c}(2 S) \rightarrow K \bar{K} \pi\right)=(1.9 \pm$ $0.4 \pm 1.1) \%$ measured by $B A B A R$ [10] is far from the worldaverage value of $\mathcal{B}\left(\eta_{c}(1 S) \rightarrow K \bar{K} \pi\right)=(7.3 \pm 0.5) \%$.

Using $637 \mathrm{fb}^{-1}$ of data, Belle reported the measurement of the $\eta_{c}(1 S)$ resonance parameters in two-photon fusion based on its decays to $\eta^{\prime} \pi^{+} \pi^{-}$with $\eta^{\prime} \rightarrow \eta \pi^{+} \pi^{-}$[11]. The above considerations motivate an updated measurement of $\eta_{c}(1 S)$ parameters using the $941 \mathrm{fb}^{-1}$ Belle data set, and, additionally, an attempt to measure $\Gamma_{\gamma \gamma}$ for $\eta_{c}(2 S)$ in order to address the discrepancy between experimental data and QCD predictions for this parameter, most of which lie in the range of $1.8-5.7 \mathrm{keV}$ [12-17].

The cross sections for two-photon production of meson pairs have been calculated in perturbative QCD and

Published by the American Physical Society under the terms of the Creative Commons Attribution 4.0 International license. Further distribution of this work must maintain attribution to the author(s) and the published article's title, journal citation, and DOI. Funded by SCOAP. measured in experiments in a $W$ region near or above $3 \mathrm{GeV}$, where $W$ is the invariant mass of the two-photon system. The leading term in the QCD calculation [18-20] of the cross section predicts a $1 /\left(W^{6} \sin ^{4} \theta\right)$ dependence for a charged-meson pair and a $1 / W^{10}$ dependence and modeldependent angular distribution for a neutral-meson pair. Here, $\theta$ is the scattering angle of a final-state particle in the two-photon CM frame. The handbag model [21] gives the transition amplitude describing energy dependence and predicts a $1 / \sin ^{4} \theta$ angular distribution for both chargedand neutral-meson pairs for large $W$. The Belle results for the cross sections [22] show that the angular distributions for the charged-meson pairs, $\gamma \gamma \rightarrow \pi^{+} \pi^{-}, K^{+} K^{-}$, agree well with the $1 / \sin ^{4} \theta$ expectation, while those for the neutral-meson pairs, $\gamma \gamma \rightarrow \pi^{0} \pi^{0}, K_{S}^{0} K_{S}^{0}, \eta \pi^{0}$ and $\eta \eta$, exhibit more complicated angular behavior. The measured exponent $n$ in the energy dependence $1 / W^{n}$ for both charged- and neutralmeson pairs is found to lie between 7.3 and 11 with a relative error of $7 \%-20 \%$. Further study with improved precision in both experiment and QCD predictions at higher $W$ mass would provide more sensitive comparisons. There is no specific QCD prediction for the two-photon production of either the pseudoscalar-tensor meson pair $\eta^{\prime} f_{2}(1270)$ or the three-body final state $\eta^{\prime} \pi^{+} \pi^{-}$. Our results for the production of these two- and three-body final states would, thus, provide new information to validate QCD models.

In this paper, we report the updated measurement of the $\eta_{c}(1 S)$ parameters with the most Belle data sample of $941 \mathrm{fb}^{-1}$, the observation of an $\eta_{c}(2 S)$ signal with its decays to $\eta^{\prime} \pi^{+} \pi^{-}$for the first time, the measurement of the product of the two-photon width of $\eta_{c}(2 S)$ and its branching fraction to $\eta^{\prime} \pi^{+} \pi^{-}$and the measurement of nonresonant production of $\eta^{\prime} \pi^{+} \pi^{-}$with $\eta^{\prime} \rightarrow \eta \pi^{+} \pi^{-}$decay via two-photon collisions.

\section{DETECTOR AND MONTE CARLO SIMULATION}

The Belle detector is a large-solid-angle magnetic spectrometer that consists of a silicon vertex detector, a 50-layer central drift chamber, an array of aerogel threshold 
Cherenkov counters, a barrel-like arrangement of time-offlight scintillation counters, and an electromagnetic calorimeter (ECL) comprised of $\mathrm{CsI}(\mathrm{Tl})$ crystals located inside a superconducting solenoid coil that provides a $1.5 \mathrm{~T}$ magnetic field. An iron flux-return located outside of the coil is instrumented to detect $K_{L}^{0}$ mesons and to identify muons. The detector is described in detail elsewhere [23].

We generate the two-photon process $\gamma \gamma \rightarrow \eta^{\prime} \pi^{+} \pi^{-}$using the TREPS code [24], where the $\eta^{\prime}$ decays generically according to JETSET7.3 [25]. A distribution uniform in phase space is assumed for the $\eta_{c}(1 S)$ and $\eta_{c}(2 S)$ decays to the $\eta^{\prime} \pi^{+} \pi^{-}$final state. The GEANT3-based [26] simulation package that incorporates the trigger conditions is employed for the propagation of the generated particles through the Belle detector.

\section{DATA AND EVENT SELECTION}

We use two data samples. The first is collected at the $\Upsilon(4 S)$ resonance $(\sqrt{s}=10.58 \mathrm{GeV})$ and $60 \mathrm{MeV}$ below it with integrated luminosity $L_{\text {int, } 4 \mathrm{~S}}=792 \mathrm{fb}^{-1}$, while the other is recorded near the $\Upsilon(5 S)$ resonance $(\sqrt{s}=10.88 \mathrm{GeV})$ with $L_{\text {int }, 5 \mathrm{~S}}=149 \mathrm{fb}^{-1}$. When combining the data in this analysis, a slight dependence of the two-photon cross section on $e^{+} e^{-}$center-of-mass energy is taken into account, as described in Sec. IV.

Two $\eta^{\prime}$ decay modes, $\eta^{\prime} \rightarrow \eta \pi^{+} \pi^{-}$with $\eta \rightarrow \gamma \gamma$ and $\eta^{\prime} \rightarrow$ $\gamma \rho$ including nonresonant $\pi^{+} \pi^{-}$(denoted as $\eta \pi \pi$ and $\gamma \rho$, respectively), are included in the reconstruction of the $\eta^{\prime}$ meson in the $\eta^{\prime} \pi^{+} \pi^{-}$final state.

\section{A. Selection criteria}

At least one neutral cluster and exactly four charged tracks with zero net charge are required in each event. The candidate photons are neutral clusters in the ECL that have an energy deposit greater than $100 \mathrm{MeV}$ and are unmatched with any charged tracks. To suppress background photons from $\pi^{0}\left(\pi^{0}\right.$ or $\left.\eta\right)$ decays for the $\eta \pi \pi(\gamma \rho)$ mode, any photon that, in combination with another photon in the event has an invariant mass within the $\pi^{0}\left(\pi^{0}\right.$ or $\left.\eta\right)$ window $\mid M_{\gamma \gamma}-$ $m_{\pi^{0}} \mid<0.018 \mathrm{GeV} / c^{2} \quad\left(\left|M_{\gamma \gamma}-m_{\pi^{0}}\right|<0.020 \mathrm{GeV} / c^{2}\right.$ or $\left.\left|M_{\gamma \gamma}-m_{\eta}\right|<0.024 \mathrm{GeV} / c^{2}\right)$ is excluded. Events with an identified kaon $\left(K^{ \pm}\right.$or $\left.K_{S}^{0} \rightarrow \pi^{+} \pi^{-}\right)$or proton are vetoed. Charged pion, kaon and proton identification strategies and criteria for the both $\eta \pi \pi$ and $\gamma \rho$ modes, as well as the event selection criteria for the $\eta \pi \pi$ mode, are the same as those used in Ref. [11] except for the requirement on the transverse momentum $\left|\Sigma p_{t}^{*}\right|$ (see Sec. III B). Here, $\left|\Sigma p_{t}^{*}\right|$ is the absolute value of the vector sum of the transverse momenta of the $\eta^{\prime}$, $\pi^{+}$, and $\pi^{-}$in the $e^{+} e^{-}$center-of-mass system. To improve the momentum resolution of the $\eta^{\prime}$, two separate fits to the $\eta^{\prime}$ are applied, one with a constrained vertex and the other with a constrained mass.

For the $\eta \pi \pi$ mode, the $\eta$ is reconstructed via its twophoton decay mode, where the two-photon invariant mass is in the window $M_{\gamma \gamma} \in[0.524,0.572] \mathrm{GeV} / c^{2}( \pm 2 \sigma$ of the nominal $\eta$ mass). The $\eta^{\prime}$ candidate is reconstructed from the $\eta$ candidate and the $\pi^{+} \pi^{-}$track pair that has an invariant mass within $M_{\eta \pi^{+} \pi^{-}} \in[0.951,0.963] \mathrm{GeV} / c^{2}( \pm 2 \sigma$ of the nominal $\eta^{\prime}$ mass).

For the $\gamma \rho$ mode, the event contains one photon and two $\pi^{+} \pi^{-}$pairs. The $\eta^{\prime}$ candidates are reconstructed with one photon candidate and a $\rho^{0}$ candidate comprised of a $\pi^{+} \pi^{-}$ pair whose invariant mass lies within the $\rho^{0}$ signal region $\left|M_{\pi^{+} \pi^{-}}-m_{\rho^{0}}\right|<0.18 \mathrm{GeV} / c^{2}$. Finally, the photon and $\rho^{0}$ candidate must satisfy $M_{\gamma \rho} \in[0.942,0.974] \mathrm{GeV} / c^{2}( \pm 2 \sigma$ of the nominal $\eta^{\prime}$ mass).

For both the $\eta \pi \pi$ and $\gamma \rho$ modes, we reconstruct $\eta^{\prime} \pi^{+} \pi^{-}$ candidates by combining the $\eta^{\prime}$ with the remaining $\pi^{+} \pi^{-}$pair, which must satisfy a vertex-constrained fit. For multicandidate events, the candidate with the smallest $\chi^{2}$ from the $\eta^{\prime}$ mass-constrained fit is selected. For $\eta^{\prime} \pi^{+} \pi^{-}$combinations with an invariant mass of $W=2.98(3.64) \mathrm{GeV} / c^{2}$, we find that $8.2 \%$ (7.3\%) of the signal Monte Carlo (MC) events have more than one candidate per event for the $\eta \pi \pi$ mode and $15 \%$ $(9.8 \%)$ for the $\gamma \rho$ mode, from which the correct candidate is selected $94 \%$ (98\%) for the $\eta \pi \pi$ mode and $88 \%(89 \%)$ for the $\gamma \rho$ mode. The sum of the ECL cluster energies in the laboratory system and the scalar sum of the absolute momenta for all charged and neutral tracks in the laboratory system for the $\eta^{\prime} \pi^{+} \pi^{-}$system must satisfy $E_{\text {sum }}<4.5 \mathrm{GeV}$ and $P_{\text {sum }}<5.5 \mathrm{GeV} / c$ to further suppress background events produced via $e^{+} e^{-} \rightarrow q \bar{q}$ with or without radiative photons.

\section{B. Optimization for the $\left|\Sigma p_{t}^{*}\right|$ requirement}

The prominent feature for the events from an untagged two-photon process in $e^{+} e^{-}$collisions is that they tend to carry small transverse momentum. Therefore, a $\left|\Sigma p_{t}^{*}\right|$ requirement allows significant background reduction. The $\left|\Sigma p_{t}^{*}\right|$ distributions for the $\eta \pi \pi$ and $\gamma \rho$ modes in the signal regions of $W \in[2.90,3.06] \mathrm{GeV}$ for $\eta_{c}(1 S)$ and $W \in[3.60,3.68] \mathrm{GeV}$ for $\eta_{c}(2 S)$ are shown in Fig. 1.

The $\left|\Sigma p_{t}^{*}\right|$ requirement for selection of the $\eta^{\prime} \pi^{+} \pi^{-}$ candidates from both the $\eta_{c}(1 S)$ and $\eta_{c}(2 S)$ decays is optimized using signal and background MC samples. The $\eta_{c}$ signal and the background are described by a relativistic Breit-Wigner function [see Eq. (1) in Sec. IV] and the exponential of a third-order polynomial, respectively. The background shape in the $\eta_{c}$ signal region is determined from the fit to the sideband data and normalized. The requirement on $\left|\Sigma p_{t}^{*}\right|$ is determined by maximizing the value of $s / \sqrt{s+b}$ for both $\eta \pi \pi$ and $\gamma \rho$ modes, where $s$ is the $\eta_{c}$ signal yield and $b$ is background yield in the $\eta_{c}$ signal region. We find the best $\left|\Sigma p_{t}^{*}\right|$ requirements, which are close to each other in the two $\eta_{c}$ mass regions, to be $\left|\Sigma p_{t}^{*}\right|<0.15 \mathrm{GeV} / c$ for the $\eta \pi \pi$ mode and $\left|\Sigma p_{t}^{*}\right|<0.03 \mathrm{GeV} / c$ for the $\gamma \rho$ mode. We find that these values are stable in the range of the expected signal yield based on the previous measurement [11] for $\eta_{c}(1 S)$ and 

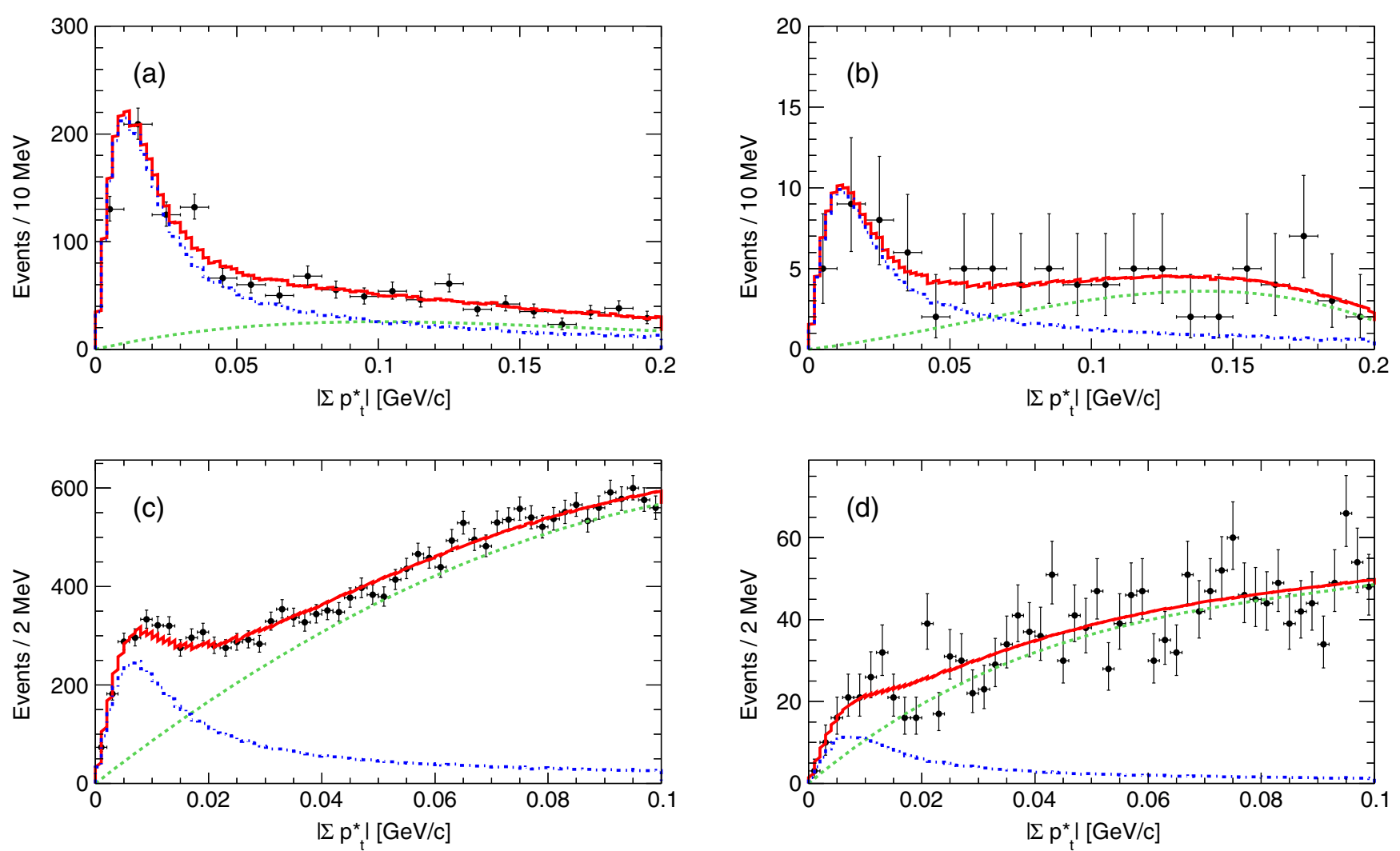

FIG. 1. The $\left|\Sigma p_{t}^{*}\right|$ distributions in the $\eta_{c}(1 S)\left[\eta_{c}(2 S)\right]$ signal region for (a) [(b)] the $\eta \pi \pi$ mode and (c) [(d)] the $\gamma \rho$ mode. The solid points with error bars are data. The solid red line is the fit; the blue dashed-dot and green dashed lines, respectively, show the signal in $\mathrm{MC}$ and the background in data.

an assumption of theoretical expectation for $\eta_{c}(2 S)$ [27]. We employ the $\left|\Sigma p_{t}^{*}\right|$ requirement values optimized for $\eta_{c}(1 S)$ to look also for the $\eta_{c}(2 S)$ in both $\eta \pi \pi$ and $\gamma \rho$ modes.

The invariant mass distributions for the candidates of the $\eta^{\prime}$ and that of the $\eta^{\prime} \pi^{+} \pi^{-}$in the $\eta \pi \pi$ and $\gamma \rho$ modes are shown in Fig. 2 and Fig. 3, respectively. In addition to the prominent $\eta_{c}(1 S)$ signal, an evident enhancement in the mass region near $3.64 \mathrm{GeV} / c^{2}$ is seen in both modes.

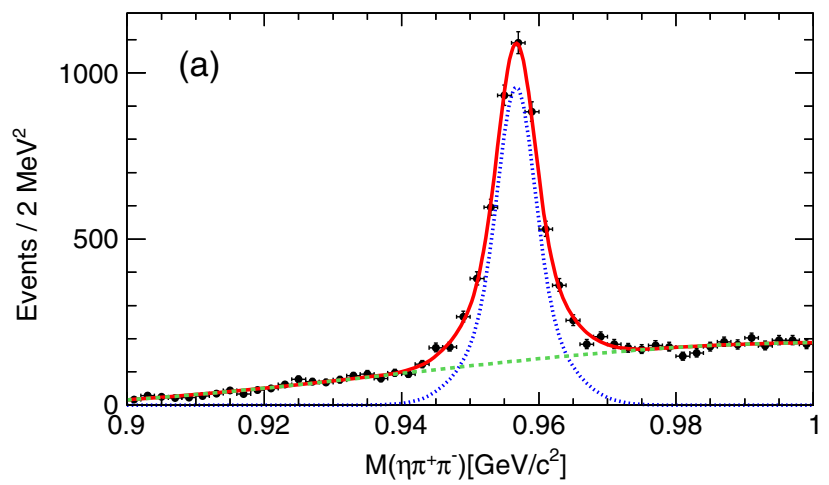

\section{FITTING FOR $\boldsymbol{\eta}_{c}(1 S)$ AND $\boldsymbol{\eta}_{c}(2 S)$}

The probability density function $f_{s}(W)$ for the resonance $R$ is a Breit-Wigner function [28,29] $f_{\mathrm{BW}}(W)$ convolved with a mass-resolution function $R_{\mathrm{ICB}}$ after corrections for the detection efficiency $\epsilon_{i}(W)$ and the two-photon luminosity function $d L_{\gamma \gamma} / d W$ :

$$
f_{\mathrm{s}}(W)=f_{\mathrm{BW}}(W) \frac{d L_{\gamma \gamma}(W)}{d W} \epsilon_{i}(W) \otimes R_{\mathrm{ICB}}(W)
$$

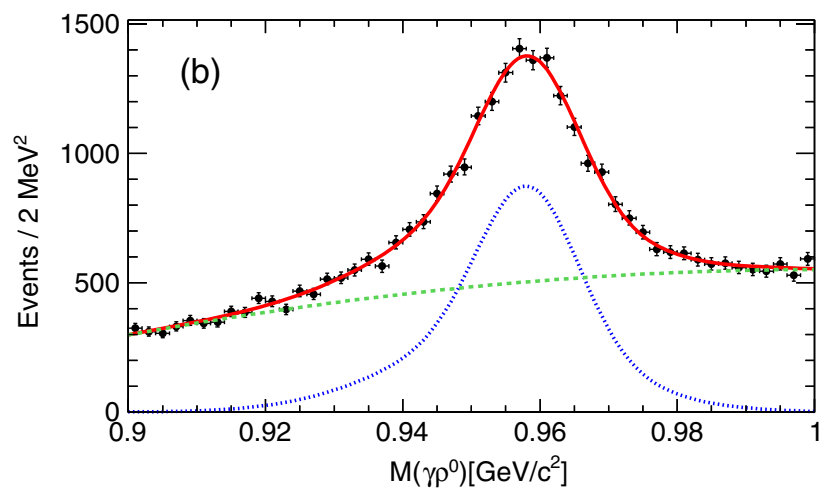

FIG. 2. The invariant mass distributions of (a) $\eta \pi^{+} \pi^{-}$and $\gamma \rho^{0}$ (b) for the $\eta^{\prime} \pi^{+} \pi^{-}$candidate events. Solid red line is the fit. The blue dashed-dot and green dashed lines are the signal and background, respectively. 

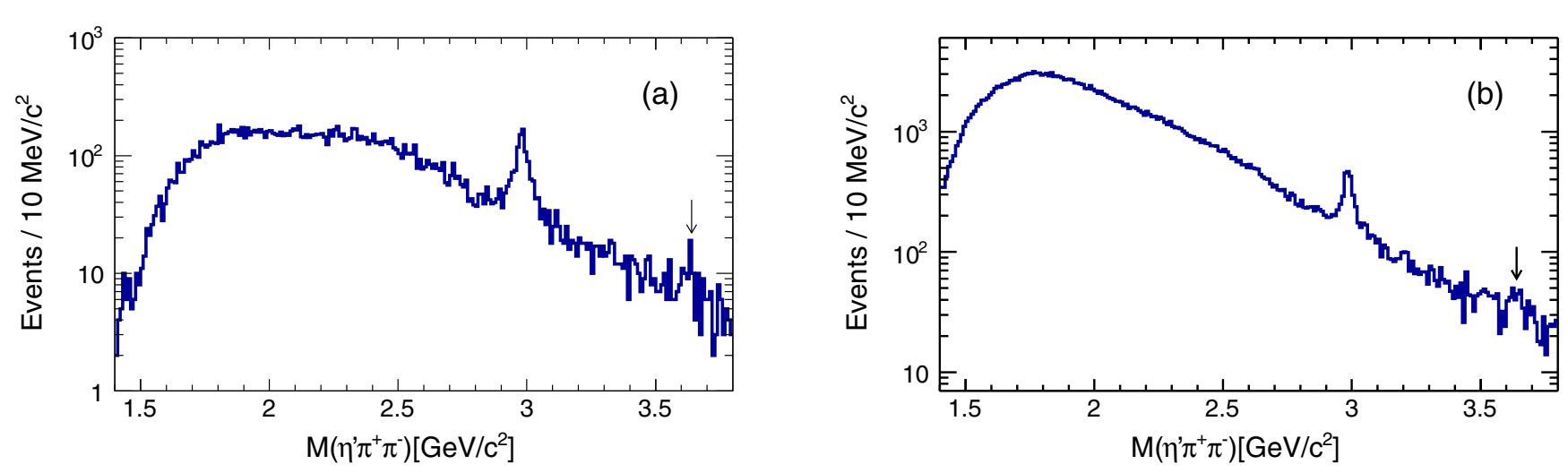

FIG. 3. The $\eta^{\prime} \pi^{+} \pi^{-}$invariant mass distribution for the candidate events with $\eta^{\prime}$ decays to (a) $\eta \pi^{+} \pi^{-}$and (b) $\gamma \rho$. Large $\eta_{c}(1 S)$ signal and evident excess in the $\eta_{c}(2 S)$ region (as arrow pointed) are seen.

Here, $R_{\mathrm{ICB}}$ is an improved Crystal Ball (ICB) function [30]. The efficiency factor $\epsilon_{i}(W)$ includes the branching fractions of $\eta^{\prime} \rightarrow \eta \pi^{+} \pi^{-}$with $\eta \rightarrow \gamma \gamma$ for the $\eta \pi \pi$ mode $(i=1)$ and $\eta^{\prime} \rightarrow \gamma \rho$ with $\rho \rightarrow \pi^{+} \pi^{-}$for the $\gamma \rho$ mode $(i=2)$. The number of the $\eta_{c}(1 S)$ mesons produced via the two-photon process is constrained to be equal for both modes in the simultaneous fit. The luminosity function is evaluated in the Equivalent Photon Approximation (EPA) $[28,29]$ using TREPS [24]. The efficiency for each $\eta^{\prime}$ decay mode is corrected for the dependence on beam energy in the $\Upsilon(4 S)$ and $\Upsilon(5 S)$ regions [31,32]:

$$
\epsilon=\frac{\epsilon_{4 \mathrm{~S}} L_{\mathrm{int}, 4 \mathrm{~S}}+\epsilon_{5 \mathrm{~S}} L_{\mathrm{int}, 5 \mathrm{~S}} \cdot \frac{d L_{\gamma \gamma, 5 \mathrm{~S}}}{d W} / \frac{d L_{\gamma \gamma, 4 \mathrm{~S}}}{d W}}{L_{\mathrm{int}, 4 \mathrm{~S}}+L_{\mathrm{int}, 5 \mathrm{~S}}},
$$

where $\epsilon_{4 \mathrm{~S}}\left(\epsilon_{5 \mathrm{~S}}\right)$ and $d L_{\gamma \gamma, 5 \mathrm{~S}} / d W\left(d L_{\gamma \gamma, 5 \mathrm{~S}} / d W\right)$ are the efficiency and two-photon luminosity functions, respectively, at the $\Upsilon(4 S)[\Upsilon(5 S)]$ energy.

The product of the two-photon decay width and the branching fraction for the $R \rightarrow \eta^{\prime} \pi^{+} \pi^{-}$decay is determined as

$$
\begin{aligned}
& \Gamma_{\gamma \gamma} \mathcal{B}\left(R \rightarrow \eta^{\prime} \pi^{+} \pi^{-}\right) \\
& =\frac{n_{\mathrm{obs}, i}}{L_{\mathrm{int}} \cdot \int f_{\mathrm{BW}}(W) \frac{d L_{\gamma \gamma}(W)}{d W} \epsilon_{i}(W) d W},
\end{aligned}
$$

where $n_{\mathrm{obs}, i}$ is the yield of decay mode $i$ of the resonance $R$ in the simultaneous fit, while $L_{\text {int }}$ is the integrated luminosity. Identical $W$ regions of $[2.60,3.4] \mathrm{GeV} / c^{2}$ for $\eta_{c}(1 S)$ and $[3.3,3.8] \mathrm{GeV} / c^{2}$ for $\eta_{c}(2 S)$ are chosen in the simultaneous fit for the yield and as the integral interval in the calculation of $\Gamma_{\gamma \gamma} \mathcal{B}$.

\section{A. Background estimation}

The background in the $\eta^{\prime} \pi^{+} \pi^{-}$mass spectrum for the $R$ measurement is dominated by three components: (1) nonresonant $(N R)$ events produced via two-photon collisions, which have the same $\left|\Sigma p_{t}^{*}\right|$ distribution as that of the $R$ signal; (2) the $\eta^{\prime}$ sideband $\left(\eta^{\prime}-s d b\right)$ arises from wrong combinations of $\gamma \gamma \pi^{+} \pi^{-}\left(\gamma \pi^{+} \pi^{-}\right)$for the $\eta \pi \pi(\gamma \rho)$ mode that survive the $\eta^{\prime}$ selection criteria, estimated using the events in the margins of the $\eta^{\prime}$ signal in the $\eta \pi \pi(\gamma \rho)$ invariant-mass distribution; (3) $\eta^{\prime} \pi^{+} \pi^{-}+X\left(b_{\text {any }}\right)$ events having additional particles in the event beyond the $R$ candidate. Other nonexclusive events, including those arising from initial-state radiation, are found to be negligible [11].

For the determination of the background components, two data subsamples, one with $\left|\Sigma p_{t}^{*}\right|<0.15 \mathrm{GeV} / c$ $(0.03 \mathrm{GeV} / c)$, denoted as $p_{t}$-balanced, and the other with $\left|\Sigma p_{t}^{*}\right| \in[0.17,0.2] \mathrm{GeV} / c([0.15,0.2] \mathrm{GeV} / c)$, denoted as $p_{t}$-unbalanced, for the $\eta \pi \pi(\gamma \rho)$ mode, are selected. (See Ref. [11] for the details.) The $R$ signal and $N R$ component peak in the $p_{t}$-balanced sample while the $\eta^{\prime}-s d b$ and $b_{\text {any }}$ backgrounds dominate over the signal plus $N R$ in the $p_{t^{-}}$ unbalanced sample. For the $\eta \pi \pi$ mode, the $\eta^{\prime}-s d b$ component is well estimated using the $\eta^{\prime}$ sideband, defined by $M_{\eta \pi^{+} \pi^{-}} \in$ $[0.914,0.934] \mathrm{GeV} / c^{2}$ and $\in[0.98,1.00] \mathrm{GeV} / c^{2}$. The $b_{\text {any }}$ component is determined using the events in the $p_{t^{-}}$ unbalanced sample with the $\eta^{\prime}-s d b$ contribution subtracted. Here, the assumption of the same shape in the invariant mass distribution for the $b_{\text {any }}$ component in the $p_{t}$-balanced and

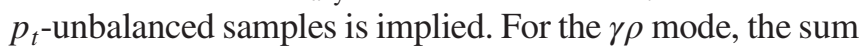
of $\eta^{\prime}-s d b$ and $b_{\text {any }}$ is determined from the events in the $p_{t^{-}}$ unbalanced sample. These two components are hard to distinguish because of peaking background in the $\gamma \rho^{0}$ invariant mass distribution, caused by the large width of the $\rho$ meson and the $\eta^{\prime}$ mass-constraint fit.

The yield and shape for the two components, $\eta^{\prime}-s d b$ and $b_{\text {any }}$, separated (combined) for the $\eta \pi \pi(\gamma \rho)$ mode, are fixed in the simultaneous fit. The exponential of a second-order polynomial is used to describe the $N R$ component with the yield and shape floating in the fit for both the $\eta \pi \pi$ and $\gamma \rho$ modes.

\section{B. Results of the $\eta_{c}(1 S)$ and $\eta_{c}(2 S)$ fits}

Simultaneous fits to the $\eta^{\prime} \pi^{+} \pi^{-}$mass spectra with the $\eta \pi \pi$ and $\gamma \rho$ modes combined are performed for both $\eta_{c}(1 S)$ 

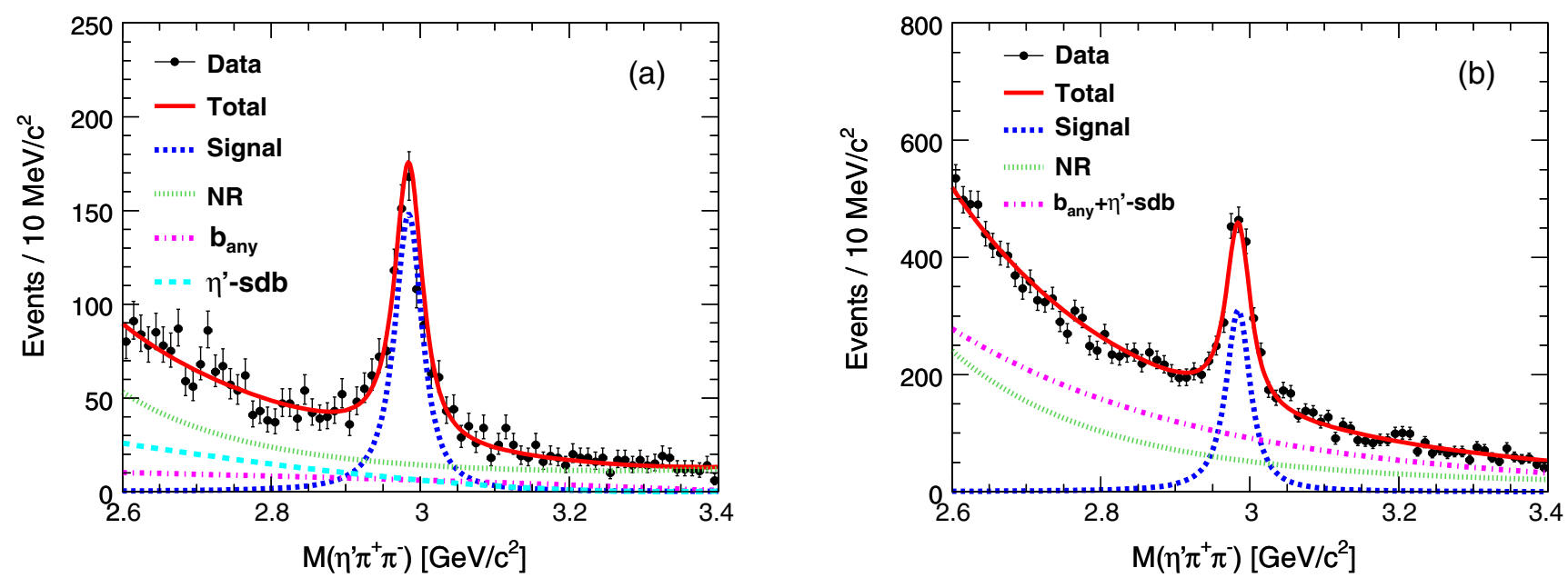

FIG. 4. The invariant mass distribution for the $\eta^{\prime} \pi^{+} \pi^{-}$candidates for (a) the $\eta \pi \pi$ mode and (b) the $\gamma \rho$ mode, in the $\eta_{c}(1 S)$ region. The dots with error bars are data. The red solid line is the fit; the blue dashed line is fitted signal for $\eta_{c}(1 S)$. The green dot, cyan long-dashed, and magenta dashed-dot lines are the $N R, \eta^{\prime}-s d b$ and $b_{\text {any }}\left(b_{\text {any }}+\eta^{\prime}-s d b\right.$ merged into the magenta dashed-dot line for the $\gamma \rho$ mode) background components, respectively.

and $\eta_{c}(2 S)$. The result of the fit for the $\eta_{c}(1 S)$ signal and background contributions are shown in Fig. 4. The $\eta_{c}(1 S)$ mass and width are determined to be $M=2984.6 \pm$ $0.7 \mathrm{MeV} / c^{2}$ and $\Gamma=30.8_{-2.2}^{+2.3} \mathrm{MeV}$, with yields of $n_{1}=$ $945_{-37}^{+38}$ for the $\eta \pi \pi$ mode and $n_{2}=1728_{-68}^{+69}$ for the $\gamma \rho$ mode.

Figure 5 shows the result of the fit for the $\eta_{c}(2 S)$ region, which results in a signal with a statistical significance of $5.5 \sigma$ and yields of $n_{1}=41_{-8}^{+9}$ for the $\eta \pi \pi$ mode and $n_{2}=65_{-13}^{+14}$ for the $\gamma \rho$ mode. The $\eta_{c}(2 S)$ mass is determined to be $M=(3635.1 \pm 3.7) \mathrm{MeV} / c^{2}$; its width is fixed to the world-average value of $11.3 \mathrm{MeV}$ [33] in the fit. The statistical significance for the $\eta_{c}(2 S)$ signal is calculated with the $\chi^{2}$ distribution $-2 \ln \left(\mathcal{L}_{0} / \mathcal{L}_{\max }\right)$ for $N_{\text {dof }}$ degrees of freedom. Here, $\mathcal{L}_{\text {max }}$ and $\mathcal{L}_{0}$ are the maximum likelihoods of the fits with the signal yield floating and fixed to zero, respectively, and $N_{\text {dof }}=2$ is the difference in the number of floating parameters between the nominal fit and the latter fit.

From Eq. (3), with the fitted signal yields as input, the product of the two-photon decay width and the branching fraction for the $\eta_{c}(1 S)$ and $\eta_{c}(2 S)$ are calculated to be $\Gamma_{\gamma \gamma} \mathcal{B}\left(\eta^{\prime} \pi^{+} \pi^{-}\right)=(65.4 \pm 2.6) \mathrm{eV}$ and $\left(5.6_{-1.1}^{+1.2}\right) \mathrm{eV}$, respectively. The fit results for the $\eta_{c}(1 S)$ and $\eta_{c}(2 S)$ are summarized in Table I.
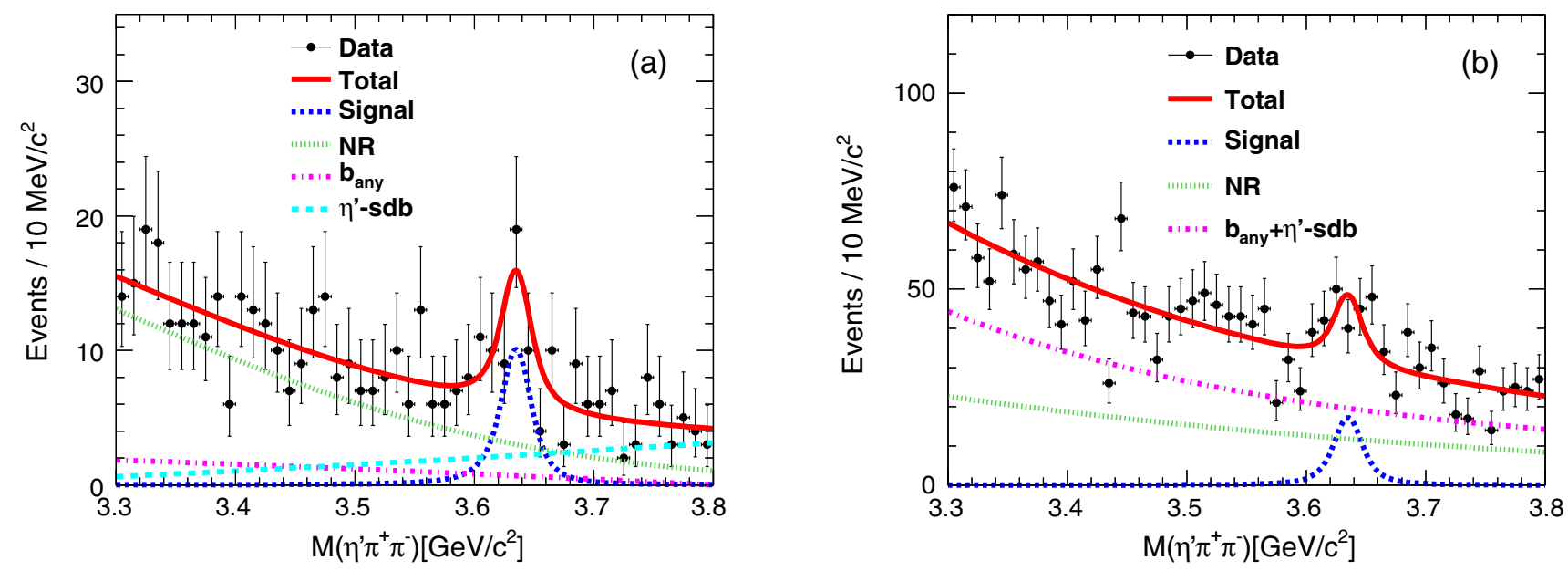

FIG. 5. The invariant mass distribution for the $\eta^{\prime} \pi^{+} \pi^{-}$candidates for (a) the $\eta \pi \pi$ mode and (b) the $\gamma \rho$ mode, in the $\eta_{c}(2 S)$ region. The dots with error bars are data. The red solid line is the fit; the blue dashed line is fitted signal for $\eta_{c}(2 S)$. The green dot, cyan long-dashed, and magenta dashed-dot lines are the $N R, \eta^{\prime}-s d b$ and $b_{\text {any }}\left(b_{\text {any }}+\eta^{\prime}-s d b\right.$ merged into the magenta dashed-dot line for the $\gamma \rho$ mode) background components, respectively. 
TABLE I. Summary of the results for the $\eta_{c}(1 S)$ and $\eta_{c}(2 S): n_{s}$ is the yield; $M$ and $\Gamma$ are the mass and width; $\Gamma_{\gamma \gamma} \mathcal{B}$ is the product of the two-photon decay width and the branching fraction for $\eta_{c} \rightarrow \eta^{\prime} \pi^{+} \pi^{-}$. The first error is statistical, and the second is systematic.

\begin{tabular}{lccccc}
\hline \hline & \multicolumn{2}{c}{$\eta_{c}(1 S)$} & & \multicolumn{2}{c}{$\eta_{c}(2 S)$} \\
\cline { 2 - 3 } \cline { 5 - 5 } & $\gamma \rho$ & $\eta \pi^{+} \pi^{-}$ & & $\gamma \rho$ & $\eta \pi^{+} \pi^{-}$ \\
\cline { 2 - 3 }$n_{s}$ & $1728_{-68}^{+69}$ & $945_{-37}^{+38}$ & & $65_{-13}^{+14}$ & $41_{-8}^{+9}$ \\
\hline$M\left(\mathrm{MeV} / c^{2}\right)$ & $2984.6 \pm 0.7 \pm 2.2$ & & $3635.1 \pm 3.7 \pm 2.9$ \\
$\Gamma(\mathrm{MeV})$ & $30.8_{-2.2}^{+2.3} \pm 2.5$ & & $11.3[$ fixed] \\
$\Gamma_{\gamma \gamma} \mathcal{B}(\mathrm{eV})$ & $65.4 \pm 2.6 \pm 7.8$ & & \multicolumn{2}{c}{$5.6_{-1.1}^{+1.2} \pm 1.1$} \\
\hline \hline
\end{tabular}

\section{Systematic uncertainties}

The systematic uncertainties are summarized in Table II. We estimate the uncertainty in the trigger efficiency using signal MC events. The differences between the two efficiencies with and without simulation of the trigger conditions are evaluated to be $0.5 \%(0.6 \%)$ for $\eta_{c}(1 S)$ $\left(\eta_{c}(2 S)\right)$ in the $\gamma \rho$ mode, and $1.4 \%$ for both $\eta_{c}$ mesons in the $\eta \pi \pi$ mode. The contribution to the systematic uncertainty arising from pion identification is studied using an inclusive $D^{*}$ sample. The uncertainties of pion identification are

TABLE II. Summary of systematic uncertainty contributions to the $\Gamma_{\gamma \gamma} \mathcal{B}$, mass and width for $\eta_{c}(1 S), \eta_{c}(2 S)$ in the fit with $\gamma \rho$ and $\eta \pi^{+} \pi^{-}$modes combined.

\begin{tabular}{|c|c|c|}
\hline \multicolumn{3}{|c|}{$\Delta\left(\Gamma_{\gamma \gamma} \mathcal{B}\right) /\left(\Gamma_{\gamma \gamma} \mathcal{B}\right)(\%)$} \\
\hline Source & $\eta_{c}(1 S)$ & $\eta_{c}(2 S)$ \\
\hline Trigger efficiency & 0.9 & 1.0 \\
\hline$\pi^{ \pm}$identification efficiency & 1.7 & 2.1 \\
\hline$\left|\Sigma p_{t}^{*}\right|$ & 1.5 & 9.8 \\
\hline Background shape & 2.3 & 9.2 \\
\hline$\eta-s d b$ and $b_{\text {any }}$ & 2.5 & 4.8 \\
\hline$\pi^{0}$-veto & 2.4 & 2.2 \\
\hline$\eta_{c}(2 S)$ width error & $\cdots$ & 8.8 \\
\hline$\eta$ reconstruction efficiency & \multicolumn{2}{|c|}{4.9} \\
\hline Track reconstruction efficiency & \multicolumn{2}{|c|}{5.5} \\
\hline Run dependence & \multicolumn{2}{|c|}{3} \\
\hline Two-photon luminosity & \multicolumn{2}{|c|}{5} \\
\hline PHSP assumption & \multicolumn{2}{|c|}{6} \\
\hline Total & 12 & 20 \\
\hline \multicolumn{3}{|l|}{$\Delta M\left(\mathrm{MeV} / c^{2}\right)$} \\
\hline Mass scale & 2.1 & 2.6 \\
\hline$\left|\Sigma p_{t}^{*}\right|$ & 0.1 & 1.1 \\
\hline Background shape & 0.7 & 0.4 \\
\hline$\eta_{c}(2 S)$ width error & $\cdots$ & 0.1 \\
\hline Total & 2.2 & 2.9 \\
\hline \multicolumn{3}{|l|}{$\Delta \Gamma(\mathrm{MeV})$} \\
\hline Mass resolution & 1.2 & $\cdots$ \\
\hline$\left|\Sigma p_{t}^{*}\right|$ & 0.7 & $\cdots$ \\
\hline Background shape & 2.1 & $\ldots$ \\
\hline Total & 2.5 & $\ldots$ \\
\hline
\end{tabular}

found to be $1.8 \%(2.3 \%)$ in the $\gamma \rho$ mode and $1.5 \%(1.8 \%)$ in the $\eta \pi \pi$ mode for $\eta_{c}(1 S)\left[\eta_{c}(2 S)\right]$. The averaged values of deviations in the yield, mass, and width between the two simultaneous fits, with the $\left|\Sigma p_{t}^{*}\right|$ requirement changed by $\pm 0.01 \mathrm{GeV} / c$ in the $\gamma \rho$ mode and by $\pm 0.02 \mathrm{GeV} / c$ in the $\eta \pi \pi$ mode, are treated as systematic uncertainties.

Two methods are applied to evaluate the systematic uncertainty related to the uncertainty in the $N R$ background shape: (1) changing the mass window size in the fit and (2) altering the fit function for the background-shape description. The difference between the average values of the two fit yields calculated by changing the mass window width by $\pm 100 \mathrm{MeV} / c^{2}$ is regarded as systematic uncertainty: we find $2.3 \%(9.0 \%)$ in the $\gamma \rho$ mode and $2.2 \%$ $(9.5 \%)$ in the $\eta \pi \pi$ mode for $\eta_{c}(1 S)\left(\eta_{c}(2 S)\right)$. The contribution to the uncertainty in the fit yield estimated by varying the order of the polynomial function is found to be minor and thus is neglected.

The uncertainty in the determination of the $\eta^{\prime}-s d b$ and $b_{\text {any }}$ backgrounds is estimated with changes in the $\eta^{\prime}-s d b$ window size by $\pm 0.01 \mathrm{GeV} / c^{2}$. The resulting difference in yields is evaluated to be $2.5 \%$ for $\eta_{c}(1 S)$ and $4.8 \%$ for $\eta_{c}(2 S)$ and is treated as the uncertainty.

The uncertainty from the $\pi^{0}$-veto is estimated as the difference in efficiency with and without the $\pi^{0}$-veto. The uncertainties for the $\eta$ reconstruction efficiency are studied using an inclusive $\eta$ sample, and its deviation from the MC simulation plus its error in quadrature is $4.9 \%$. The systematic uncertainties related to charged track reconstruction efficiency, luminosity function calculation, and experimental-conditions dependence are studied via charmonium decay to four charged mesons $[7,8]$. The evolution of the background conditions over time adds an additional uncertainty of $3 \%$ in the yield determination. The accuracy of the two-photon luminosity is estimated to be 5\% including the uncertainties from radiative corrections $(2 \%)$, the uncertainty from the form-factor effect (2\%), and the error of the integrated luminosity $(1.36 \%)$.

The efficiency for the $\eta^{\prime} \pi^{+} \pi^{-}$events is determined with the MC sample generated with $\eta_{c}(1 S)$ decays to three-body $\eta^{\prime} \pi^{+} \pi^{-}$according to phase space distribution. Possible intermediate states in $\eta_{c}(1 S)$ decays are checked in data. Figure 6 shows the Dalitz plots for the $\eta^{\prime} \pi^{+} \pi^{-}$ events selected in the $\eta_{c}(1 S)$ signal window of $[2.90,3.06] \mathrm{GeV} / c^{2}$ and sideband region of $[2.60,2.81] \cup$ $[3.15,3.36] \mathrm{GeV} / c^{2}$ (denoted as $s d b$ ) in the $\eta \pi \pi$ mode. Figures 7(a) and 7(c) show the $\eta^{\prime} \pi^{+}$(charge conjugate implied, two entries per event) and $\pi^{+} \pi^{-}$invariant mass distributions for the events selected in the $\eta_{c}(1 S)$ signal and $s d b$ regions. The corresponding mass distributions after subtraction of the normalized $s d b$ background are shown in Figs. 7(b) and 7(d). Broad structures are seen in distributions of both $M\left(\eta^{\prime} \pi^{+}\right)$near $1.7 \mathrm{GeV} / c^{2}$ and $M\left(\pi^{+} \pi^{-}\right)$near $2 \mathrm{GeV} / c^{2}$. To estimate the effect on the efficiency due to the two-body intermediate states in $\eta_{c}(1 S)$ decays, a 

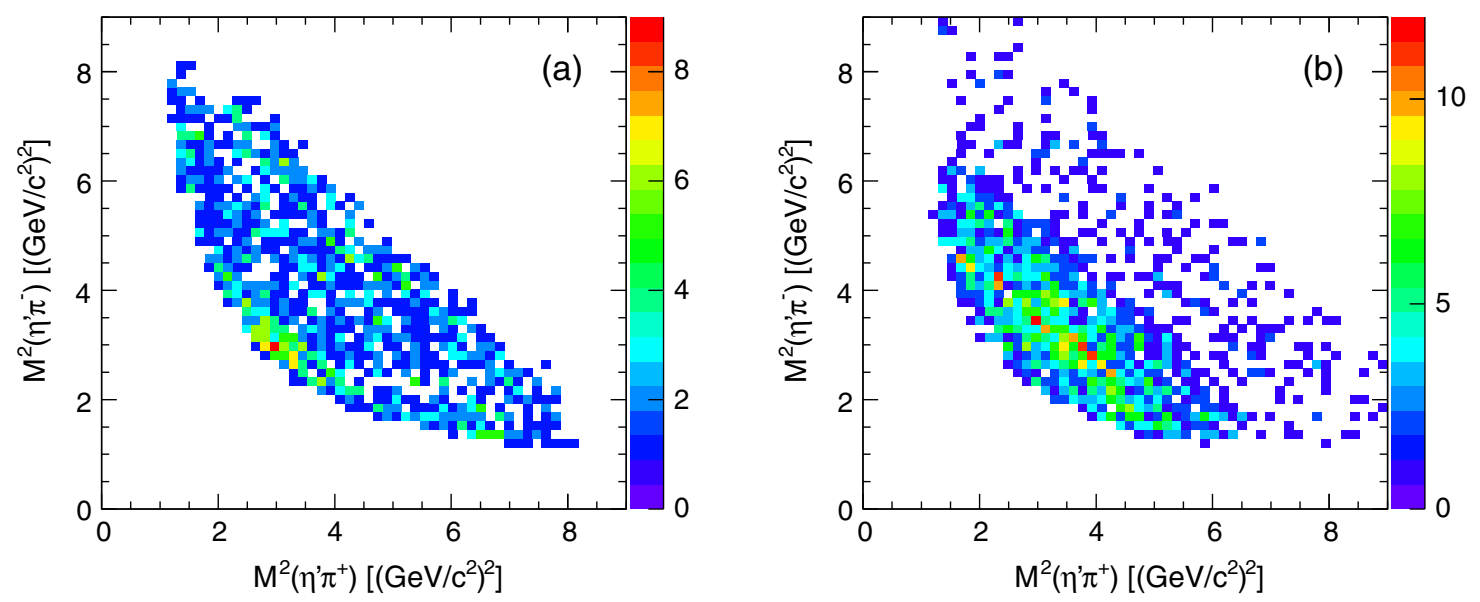

FIG. 6. The Dalitz plots for events selected in the $\eta_{c}(1 S)$ signal (a) and $s d b$ (b) regions.

possible two-body intermediate state $\eta_{c}(1 S) \rightarrow \eta^{\prime} f_{0}(2100)$ is assumed and simulated, and the averaged efficiency of this mode and the three-body phase space sample is calculated. Here, an approximately equal ratio of two yields $n_{\mathrm{s} \text {,three-body }} / n_{\mathrm{s} \text {,two-body }}$ is assumed in averaging the two modes. The relative difference in efficiencies between the phase space (PHSP) MC sample and the average efficiency is estimated to be $\Delta \epsilon_{\mathrm{avr}, \eta \pi \pi}=8.8 \%$ $\left(\Delta \epsilon_{\mathrm{avr}, \gamma \rho}=3.6 \%\right)$ for the $\eta \pi \pi(\gamma \rho)$ mode. Taking the yield-weighted mean of $\Delta \epsilon_{\mathrm{avr}, \eta \pi \pi}$ and $\Delta \epsilon_{\mathrm{avr}, \gamma \rho}$ for the $\eta \pi \pi$ and $\gamma \rho$ modes combined in the fits, the uncertainty in efficiency related to the assumption of the uniform
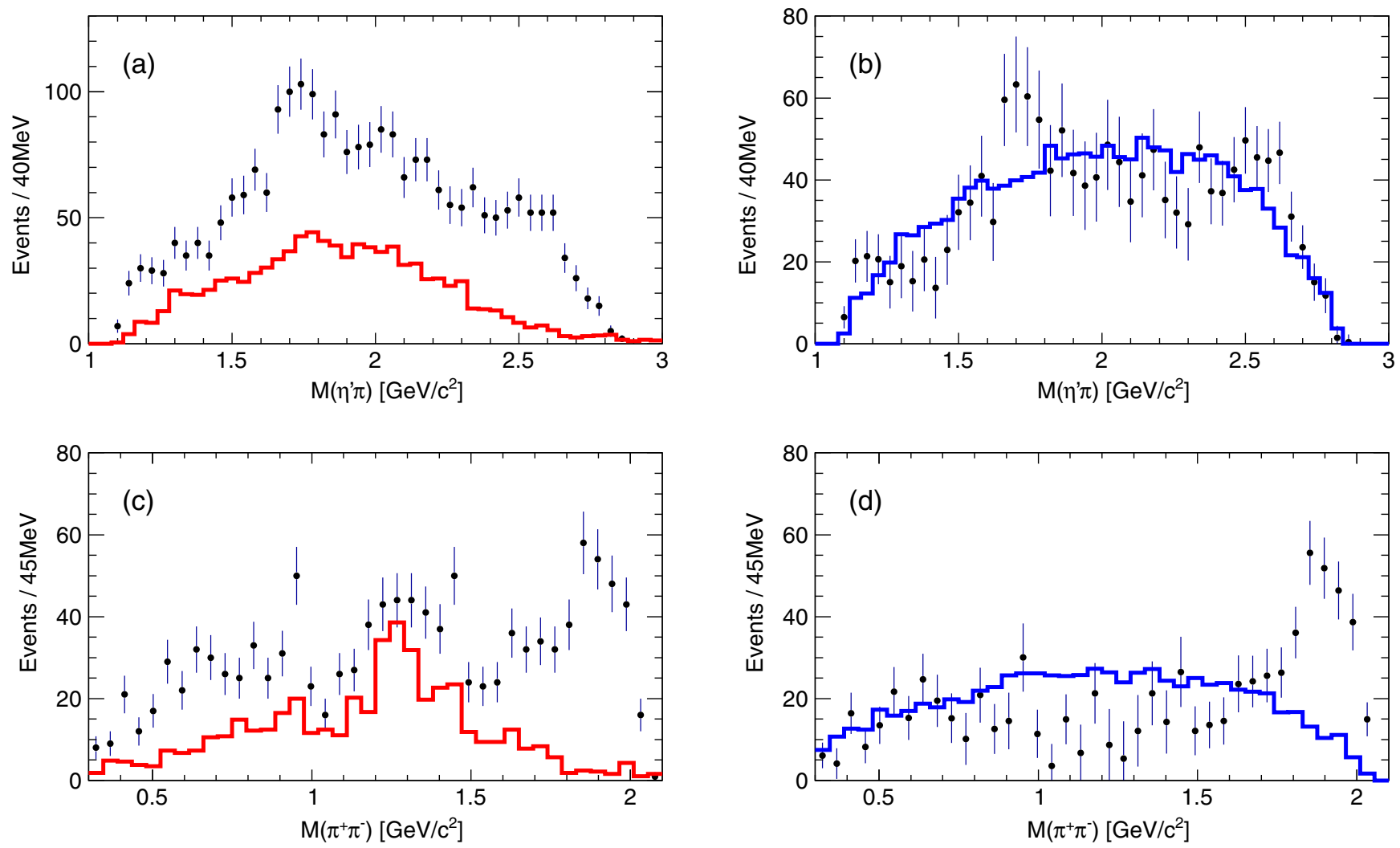

FIG. 7. (a) [(c)] The invariant mass $M\left(\eta^{\prime} \pi^{+}\right)$distributions (two entries per event) $\left[M\left(\pi^{+} \pi^{-}\right)\right.$distributions] in data for the events selected in the $\eta_{c}(1 S)$ signal region is drawn as the black solid dots with error bars. The red histogram is for the normalized $s d b$ background events. (b) [(d)] The black solid dots with error bars is the $M\left(\eta^{\prime} \pi^{+}\right)\left[M\left(\pi^{+} \pi^{-}\right)\right]$distribution in the $\eta_{c}(1 S)$ signal region in data after subtraction of the $s d b$ background and the blue histogram normalized to data is for MC events of the $\eta_{c}(1 S)$ decays to threebody final state according to PHSP distribution. 
TABLE III. Defined bin size and total number of bins in $W$ and $\left|\cos \theta^{*}\right|$ in individual $W$ ranges.

\begin{tabular}{ccc}
\hline \hline$W[\mathrm{GeV}]$ & $\Delta W \times N_{\text {bins }}[\mathrm{GeV}]$ & $\Delta\left|\cos \theta^{*}\right| \times N_{\text {bins }}$ \\
\hline $1.40-1.66$ & $0.26 \times 1$ & $0.1 \times 10$ \\
$1.66-1.82$ & $0.08 \times 2$ & $0.1 \times 10$ \\
$1.82-2.66$ & $0.04 \times 21$ & $0.1 \times 10$ \\
$2.66-3.08$ & $0.06 \times 7$ & $0.2 \times 5$ \\
$3.08-3.40$ & $0.16 \times 2$ & $0.2 \times 5$ \\
$3.40-3.80$ & $0.20 \times 2$ & $0.2 \times 5$ \\
\hline \hline
\end{tabular}

distribution in PHSP is found to be 6\%, which is added to the systematic error.

To examine the systematic uncertainty in the mass measurement for the $R \rightarrow \eta^{\prime} \pi^{+} \pi^{-}$decay, an inclusive control sample of the decay $D^{0} \rightarrow \eta^{\prime} K_{S}^{0}$ with $K_{S}^{0} \rightarrow$ $\pi^{+} \pi^{-}$is selected with a tight mass window for $\eta^{\prime}$. The $D^{0}$ mass resulting from fits to the invariant mass spectra of $\eta^{\prime} K_{S}^{0}$ is shifted from its nominal value by $1.26 \mathrm{MeV} / c^{2}$ $\left(0.93 \mathrm{MeV} / c^{2}\right)$ in the $\eta \pi \pi(\gamma \rho)$ mode. The sum of the shift and statistical error in quadrature, scaled linearly to the $\eta_{c}$ mass, is taken as the contribution of the uncertainty for the mass scale. The uncertainty in the width determination is estimated by changing the mass resolution by $\pm 1 \mathrm{MeV} / c^{2}$, and is found to be $1.2 \mathrm{MeV} / c^{2}$ for the $\eta_{c}(1 S)$. The uncertainties for the resonance mass and width coming from $\left|\Sigma p_{t}^{*}\right|$ and background shape are determined with the same method as that for the $\Gamma_{\gamma \gamma} \mathcal{B}$ measurement.

Taking the yield-weighted mean of squared uncertainty for the $\gamma \rho$ and $\eta \pi^{+} \pi^{-}$modes combined in the fits, the total systematic uncertainties in the measurements of $\Gamma_{\gamma \gamma} \mathcal{B}$, mass and width for $\eta_{c}(1 S)\left[\eta_{c}(2 S)\right]$ are calculated by adding the individual mean uncertainties in quadrature.

\section{MEASUREMENTS OF THE CROSS SECTIONS}

We utilize the data sample selected in the $\eta^{\prime} \rightarrow \eta \pi \pi$ mode to measure the nonresonant production of $\eta^{\prime} \pi^{+} \pi^{-}$final states via two-photon collisions. The cross section of $e^{+} e^{-} \rightarrow e^{+} e^{-} h$ production is expressed as

$$
\begin{aligned}
\sigma_{e^{+} e^{-} \rightarrow e^{+} e^{-} h}= & \int \sigma_{\gamma \gamma \rightarrow h}\left(W,\left|\cos \theta^{*}\right|\right) \\
& \times \frac{d L_{\gamma \gamma}(W)}{d W} d W d\left|\cos \theta^{*}\right|,
\end{aligned}
$$

where $h$ denotes one of two hadronic final states: $\eta^{\prime} \pi^{+} \pi^{-}$or $\eta^{\prime} f_{2}(1270)$. Here, $\theta^{*}$ is the angle between the $\eta^{\prime}$ momentum and the beam direction in the $\gamma \gamma$ rest frame.

The differential cross section in the measurement of the $W$ and $\left|\cos \theta^{*}\right|$ two-dimensional distribution for the finalstate particles is calculated with the formula below, accounting for the efficiencies as a function of the measured variables.

$$
\frac{d \sigma_{\gamma \gamma \rightarrow h}\left(W, \cos \theta^{*}\right)}{d\left|\cos \theta^{*}\right|}=\frac{\Delta N\left(W, \cos \theta^{*}\right) / \epsilon\left(W, \cos \theta^{*}\right)}{L_{\text {int }} \frac{d L_{\gamma \gamma}(W)}{d W} \Delta W \Delta\left|\cos \theta^{*}\right|},
$$

where the yield $\Delta N$ is extracted by fitting the $\left|\Sigma p_{t}^{*}\right|$ $\left[M\left(\pi^{+} \pi^{-}\right)\right]$distribution in a data subsample sliced in each two-dimensional bin for the $\gamma \gamma \rightarrow \eta^{\prime} \pi^{+} \pi^{-}[\gamma \gamma \rightarrow$ $\left.\eta^{\prime} f_{2}(1270)\right]$ production. The efficiency $\epsilon\left(W, \cos \theta^{*}\right)$ is evaluated using MC events for each two-dimensional bin. $L_{\text {int }}$ is the total integrated luminosity of the data and $d L_{\gamma \gamma} / d W$ is the two-photon luminosity function.

The $W$-dependent cross sections of $\gamma \gamma \rightarrow h$ are obtained by a summation over $\left|\cos \theta^{*}\right|$ bins as

$$
\sigma_{\gamma \gamma \rightarrow h}(W)=\sum_{\Delta\left|\cos \theta^{*}\right|} \frac{d \sigma_{\gamma \gamma \rightarrow h}\left(W, \cos \theta^{*}\right)}{d\left|\cos \theta^{*}\right|} \Delta\left|\cos \theta^{*}\right|
$$

\section{A. Cross sections of $\gamma \gamma \rightarrow \eta^{\prime} \pi^{+} \pi^{-}$(including $\eta^{\prime} f_{2}(1270)$ )}

We divide the $W$ distribution between 1.40 and $3.80 \mathrm{GeV}$ into 35 bins and the $\left|\cos \theta^{*}\right|$ distribution into 10 and 5 bins for the $W$ regions of 1.40 to $2.66 \mathrm{GeV}$ and 2.66 to $3.80 \mathrm{GeV}$, respectively. The defined bin size and total number of bins in $W$ and $\left|\cos \theta^{*}\right|$ are listed in the Table III. Detection

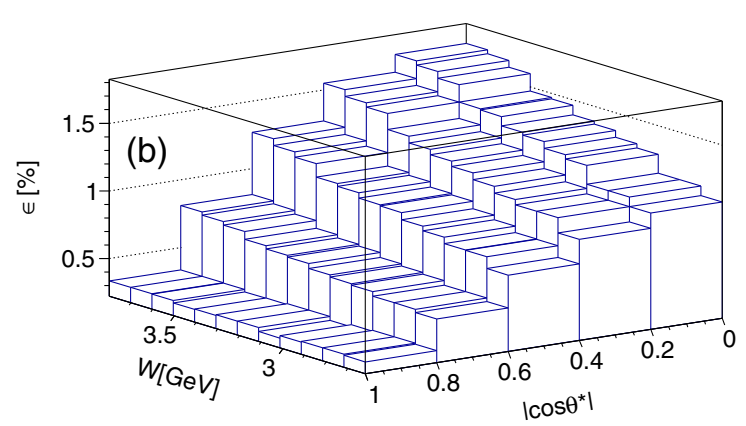

FIG. 8. Detection efficiency $\epsilon$ as a function of $W$ and $\left|\cos \theta^{*}\right|$ for $\gamma \gamma \rightarrow \eta^{\prime} \pi^{+} \pi^{-}$with the $\eta \pi^{+} \pi^{-}$mode in the regions of (a) $W \in[1.40,2.66) \mathrm{GeV}$ and (b) $W \in[2.66,3.80] \mathrm{GeV}$. 


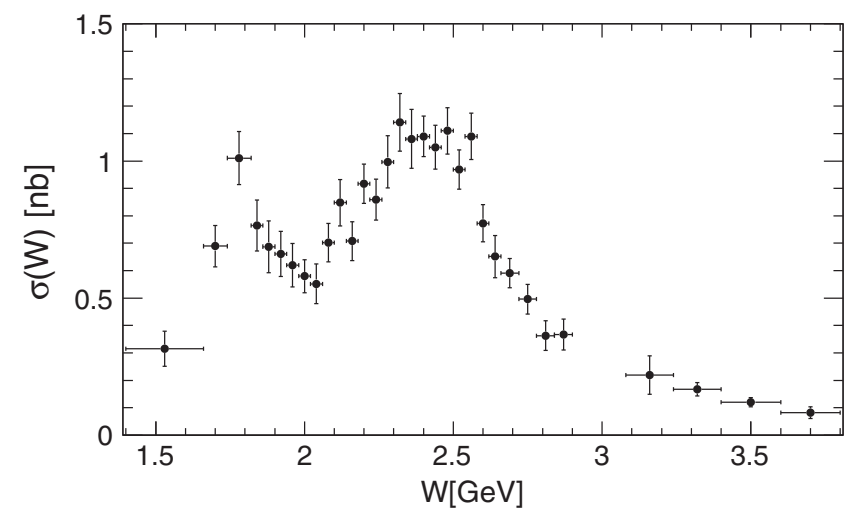

FIG. 9. Measured cross section of $\gamma \gamma \rightarrow \eta^{\prime} \pi^{+} \pi^{-}$(including $\left.\eta^{\prime} f_{2}(1270)\right)$ for the $\eta \pi \pi$ mode.

efficiencies as a function of $W$ and $\left|\cos \theta^{*}\right|$ are shown in Fig. 8. The yield $\Delta N$ in Eq. (5) is extracted by fitting the $\left|\Sigma p_{t}^{*}\right|$ distribution in data for each two-dimensional bin. For the fit, the signal shape in MC is fixed, the $\eta^{\prime}-s d b$ background in data is normalized and fixed, and the $b_{\text {any }}$ background is described by a third-order polynomial with its constant term fixed at 0 and the other parameters floating.

A background arising from $\eta^{\prime} \rightarrow \gamma \rho$ decays in the candidate events of the $\eta \pi \pi$ mode is studied using the MC sample. One photon and four charged-pion tracks in the MC event, produced for the $\gamma \rho$ mode, plus a fake photon, is wrongly chosen as an $\eta^{\prime} \pi^{+} \pi^{-}$combinatorial candidate for the $\eta \pi \pi$ mode. Here, the fake photon with low momentum is a neutral track composed of background hits or hit clusters split from charged pion tracks in the ECL. This appears as a background component because of the additional fake photon in the event; it is estimated using the premeasured cross section for $\gamma \gamma \rightarrow \eta^{\prime} \pi^{+} \pi^{-}$in data for the $\eta \pi \pi$ mode and is found to be small. The measured cross section for $\gamma \gamma \rightarrow \eta^{\prime} \pi^{+} \pi^{-}$for the $\eta \pi \pi$ mode after subtraction of this small contamination is shown in Fig. 9.

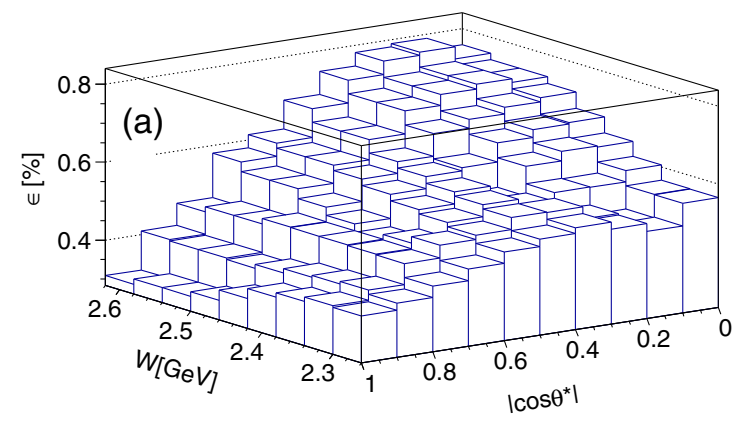

\section{B. Result for the $\gamma \gamma \rightarrow \eta^{\prime} f_{2}(1270)$ cross section measurement}

To calculate the cross section for the $\gamma \gamma \rightarrow \eta^{\prime} f_{2}(1270)$ production, we divide $W$ into 16 bins from 2.26 to $3.80 \mathrm{GeV}$, and $\left|\cos \theta^{*}\right|$ into 10 and 5 bins $\left(0<\left|\cos \theta^{*}\right|<1\right)$ for the regions of $W \in[2.26,2.62) \mathrm{GeV}$ and $[2.62,3.80] \mathrm{GeV}$, respectively. The efficiency $\epsilon$ in each two-dimensional bin, evaluated using signal MC events for $\gamma \gamma \rightarrow$ $\eta^{\prime} f_{2}(1270)$ with the phase-space distribution, is shown in Fig. 10.

The yield $\Delta N$ of $f_{2}(1270)$ in Eq. (5) is extracted by fitting the invariant mass spectrum of $\pi^{+} \pi^{-}$for the $f_{2}(1270)$ signal using the data subsample in each twodimensional bin. A broad $f_{2}(1270)$ signal in the $W$ region from 2.26 to $2.62 \mathrm{GeV}$ near threshold is described by a $D$-wave Breit-Wigner function,

$$
f_{\mathrm{BW}}=\frac{1}{\left(W^{2}-M^{2}\right)^{2}+M^{2} \Gamma^{2}} q p^{5}
$$

where $M$ and $\Gamma$ are the $f_{2}(1270)$ mass and width. The $q$ and $p$ momentum variables are, respectively, of the $f_{2}(1270)$ in the $\gamma \gamma$ rest frame and of the $\pi$ meson from the $f_{2}(1270)$ decay in the $f_{2}(1270)$ rest frame. In the fits, $\Gamma$ is fixed to the world-average value, and $M$ is fixed to the value extracted from fitting the $\pi^{+} \pi^{-}$invariant mass spectrum for the $f_{2}(1270)$ using events in the full range of $W\left(\left|\cos \theta^{*}\right|<1\right)$. The $f_{2}(1270)$ signal in the $W$ region above $2.62 \mathrm{GeV}$ is described by a normal Breit-Wigner function with both $M$ and $\Gamma$ fixed to the world-average values. We fix the fraction of the $\eta^{\prime}-s d b$ background in the fits. The combinatorial background, including non- $f_{2}(1270)$ and $b_{\text {any }}$ events, is described by a fourth-order polynomial with its parameters fixed to the values extracted from the $f_{2}(1270)$ fit for each $W$ bin.

The $W$-dependent cross section for $\gamma \gamma \rightarrow \eta^{\prime} f_{2}(1270)$ in the $\eta \pi \pi$ mode, calculated with Eq. (5), is shown in Fig. 11 and listed in Table IV. The differential cross sections in $\left|\cos \theta^{*}\right|$, averaged over $W$ bins in the three ranges

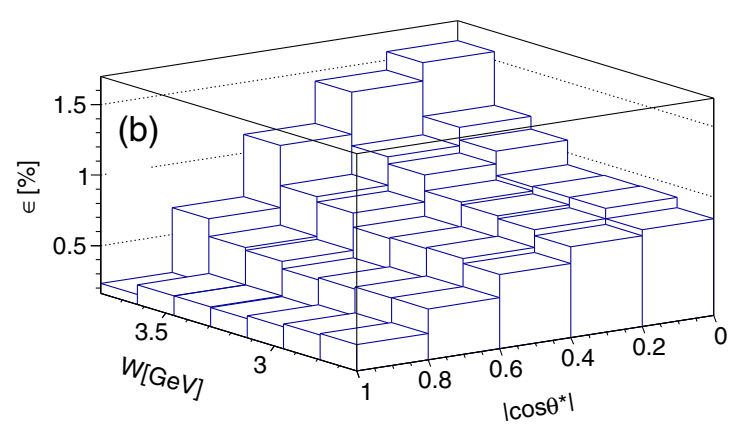

FIG. 10. Detection efficiency $\epsilon$ as a function of $W$ and $\left|\cos \theta^{*}\right|$ for $\gamma \gamma \rightarrow \eta^{\prime} f_{2}(1270)$ in the $\eta \pi \pi$ mode in the $W$ ranges of (a) $[2.26,2.62) \mathrm{GeV}$ and (b) $[2.62,3.80] \mathrm{GeV}$. 

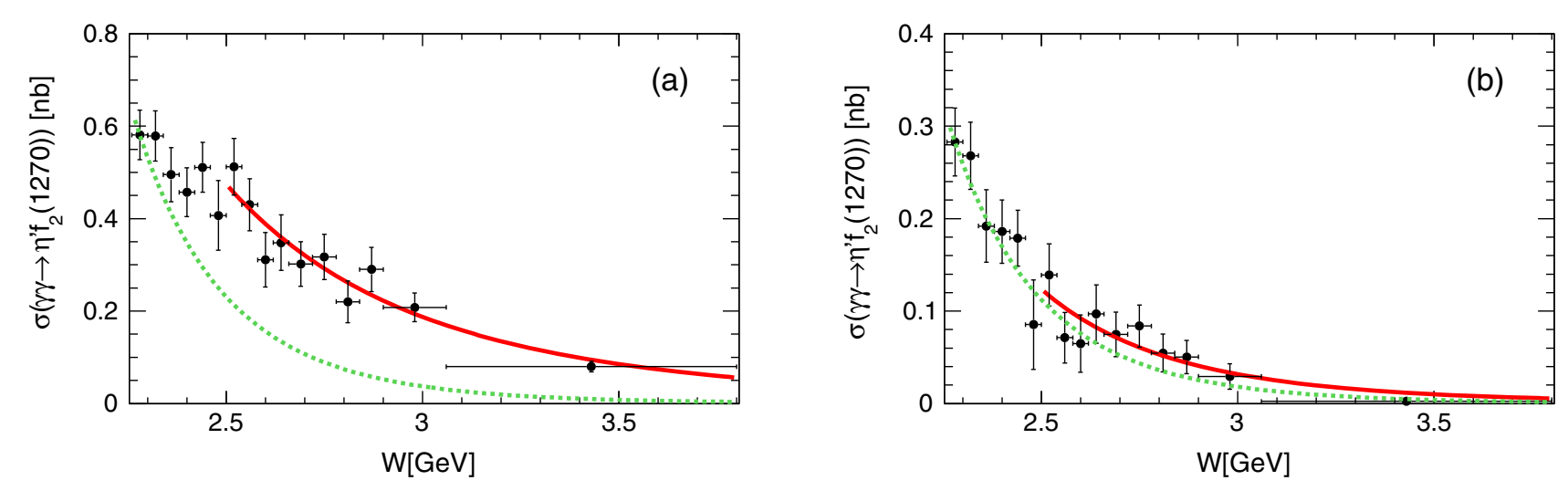

FIG. 11. Measured cross sections for $\gamma \gamma \rightarrow \eta^{\prime} f_{2}(1270)$. The black dots with statistical error bars are the data within (a) $\left|\cos \theta^{*}\right|<1$ and (b) $\left|\cos \theta^{*}\right|<0.6$. The red solid lines are fitted curves with the $W$-power index $n=5.1 \pm 1.0$ and $n=7.5 \pm 2.0$, respectively, assuming a $W$ dependence of $1 / W^{n}$. The green dashed line corresponds to the leading-term QCD prediction for neutral meson pairs $(n=10)$.

$W \in[2.26,2.50),[2.50,2.62),[2.62,3.80] \mathrm{GeV}$, are given in Fig. 12.

We assume that the $W$ and $\theta^{*}$ dependencies of the differential cross section follow the power law $\sigma \propto 1 / W^{n} \cdot \sin ^{\alpha} \theta^{*}$, which is the same as that for pseudoscalar meson pairs in the Belle data and the QCD predictions [22]. In a fit to the measured cross sections for $\gamma \gamma \rightarrow \eta^{\prime} f_{2}(1270)$ in the range of $W \in[2.5,3.8] \mathrm{GeV}$, the resulting $W$ power-law exponent is $n=7.7 \pm$ $1.5(7.5 \pm 2.0)$ for $\left|\cos \theta^{*}\right| \in[0.0,0.8](\in[0.0,0.6])$. The differential cross sections in $\left|\cos \theta^{*}\right|$ show an ascending trend in all three $W$ ranges, and its rate of increase is greater for events in the larger $W$ ranges. The complicated behavior for the angular dependence of the cross sections is seen in
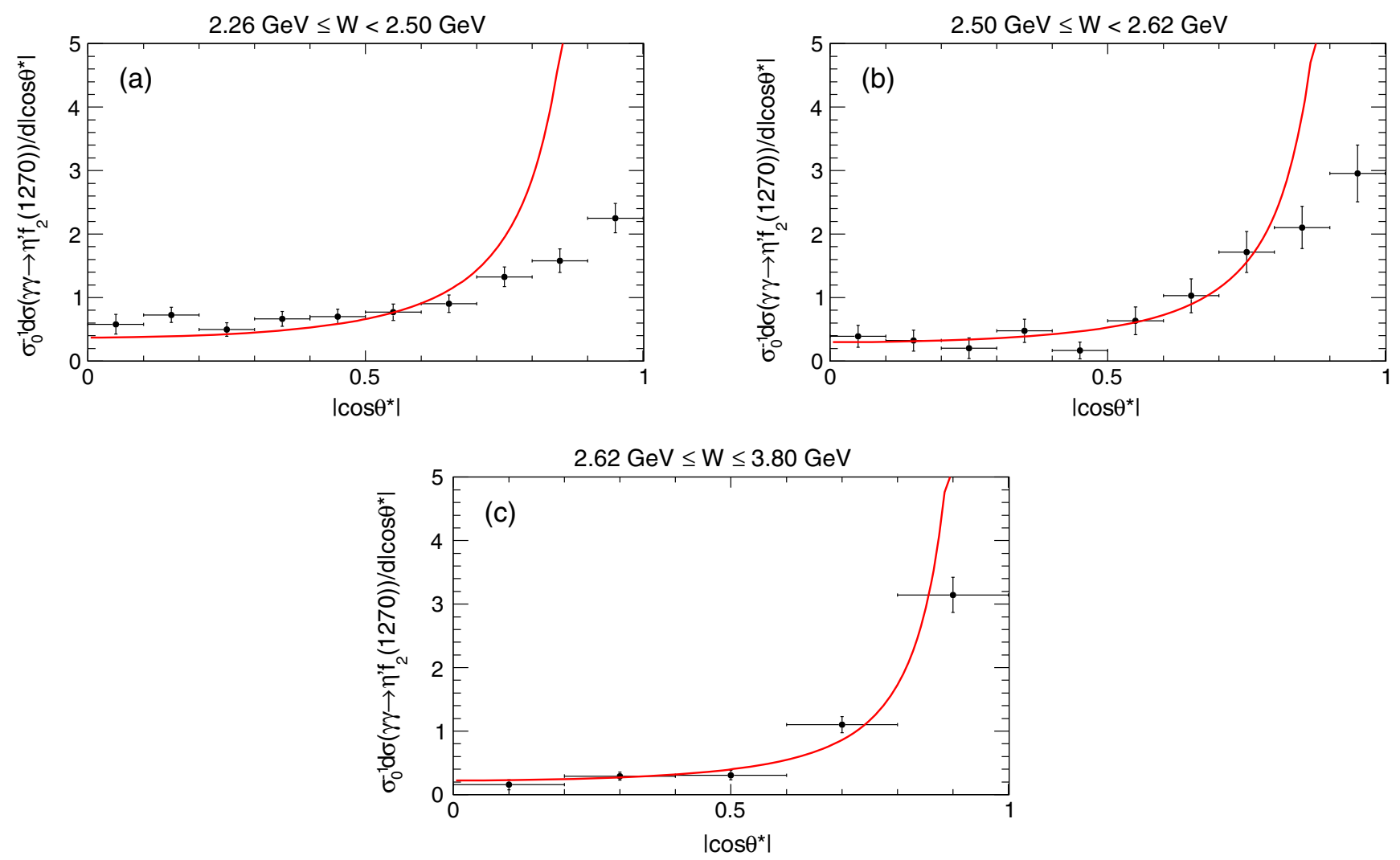

FIG. 12. Cross sections of $\gamma \gamma \rightarrow \eta^{\prime} f_{2}(1270)$ in $\left|\cos \theta^{*}\right|$ in three $W$ regions from 2.26 to $3.80 \mathrm{GeV}$. The normalizer $\sigma_{0}$ is the total cross section in the $\left|\cos \theta^{*}\right|<0.8$ region. The black solid points are the data with statistical errors. The red solid line, normalized to the data in the same angular range, follows a $1 / \sin ^{4} \theta^{*}$ behavior. 
TABLE IV. Measured cross sections as a function of $W$ within $\left|\cos \theta^{*}\right|<1$ for $\gamma \gamma \rightarrow \eta^{\prime} f_{2}(1270)$ in the $\eta \pi \pi$ mode. The first error is statistical, and the second is systematic.

\begin{tabular}{lc}
\hline \hline$W(\mathrm{GeV})$ & $\sigma\left(\gamma \gamma \rightarrow \eta^{\prime} f_{2}(1270)\right)(\mathrm{nb})$ \\
\hline $2.26-2.30$ & $0.58 \pm 0.05 \pm 0.11$ \\
$2.30-2.34$ & $0.58 \pm 0.05 \pm 0.11$ \\
$2.34-2.38$ & $0.495 \pm 0.059 \pm 0.091$ \\
$2.38-2.42$ & $0.457 \pm 0.053 \pm 0.087$ \\
$2.42-2.46$ & $0.511 \pm 0.054 \pm 0.098$ \\
$2.46-2.50$ & $0.407 \pm 0.075 \pm 0.086$ \\
$2.50-2.54$ & $0.512 \pm 0.061 \pm 0.091$ \\
$2.54-2.58$ & $0.430 \pm 0.056 \pm 0.078$ \\
$2.58-2.62$ & $0.311 \pm 0.059 \pm 0.063$ \\
$2.62-2.66$ & $0.348 \pm 0.060 \pm 0.063$ \\
$2.66-2.72$ & $0.302 \pm 0.048 \pm 0.058$ \\
$2.72-2.78$ & $0.317 \pm 0.049 \pm 0.053$ \\
$2.78-2.84$ & $0.220 \pm 0.045 \pm 0.037$ \\
$2.84-2.90$ & $0.290 \pm 0.048 \pm 0.051$ \\
$2.90-3.06$ & $0.208 \pm 0.031 \pm 0.043$ \\
$3.06-3.80$ & $0.080 \pm 0.011 \pm 0.019$ \\
\hline \hline
\end{tabular}

the range of $W<2.50 \mathrm{GeV}$ with markedly lower power for $\sin \theta^{*}$ of $\alpha<4$, while it tends to match with the power law for the ranges of $W \in[2.50,2.62]$ and $[2.62,3.80] \mathrm{GeV}$.

\section{Result for the $\gamma \gamma \rightarrow \eta^{\prime} \pi^{+} \pi^{-}$(excluding $\eta^{\prime} f_{2}(1270)$ ) cross sections}

In the left plot of Fig. 13, the measured $W$-dependent cross sections of $\gamma \gamma \rightarrow \eta^{\prime} f_{2}(1270)$ and $\gamma \gamma \rightarrow \eta^{\prime} \pi^{+} \pi^{-}$ [including $\eta^{\prime} f_{2}(1270)$ ] production are shown. The former is obtained by fitting the $\pi^{+} \pi^{-}$invariant mass spectrum for the $f_{2}(1270)$ signal and the latter is extracted in fitting the $\left|\Sigma p_{t}^{*}\right|$ distribution for the $\eta^{\prime} \pi^{+} \pi^{-}$signal. Taking the difference between the two yields in each two-dimensional bin in data as input, the cross sections of $\gamma \gamma \rightarrow \eta^{\prime} \pi^{+} \pi^{-}$production

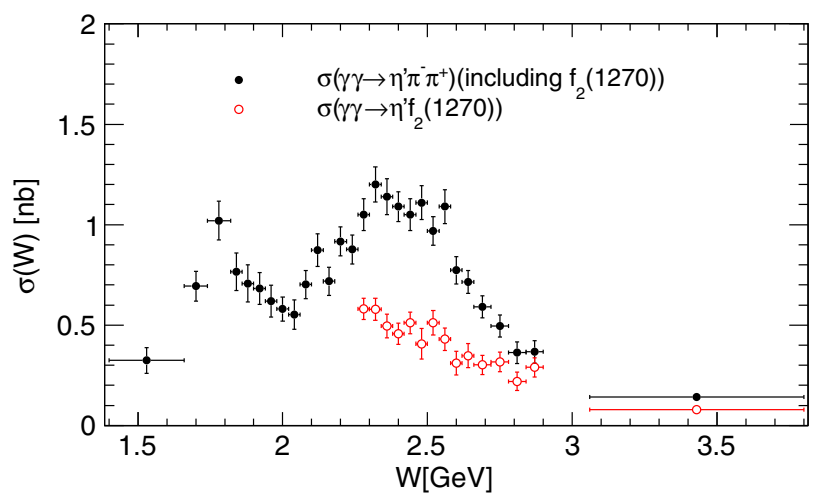

without the $\eta^{\prime} f_{2}(1270)$ contribution for the $\eta \pi \pi$ mode are calculated and shown in the right plot of Fig. 13 and summarized in Table V. Two peaking structures are evident. The one around $1.8 \mathrm{GeV}$ likely arises from the $\eta(1760)$ and $X(1835)$ decays to $\eta^{\prime} \pi^{+} \pi^{-}$[11], and the other around $2.15 \mathrm{GeV}$ is possibly due to $\gamma \gamma \rightarrow \eta^{\prime} f_{0}(980)$ production. The $\eta_{c}(1 S)$ contribution near $2.98 \mathrm{GeV}$ has been subtracted. A larger data sample is necessary in order to understand these two structures in more detail.

The differential cross section in $\left|\cos \theta^{*}\right|$ for $\gamma \gamma \rightarrow$ $\eta^{\prime} \pi^{+} \pi^{-}$production after subtracting both contributions from $\gamma \gamma \rightarrow \eta^{\prime} f_{2}(1270)$ in the $W$ region above $2.26 \mathrm{GeV}$ and $\eta_{c}(1 S)$ in the region of $W \in[2.62,3.06] \mathrm{GeV}$ is shown in Fig. 14. Nearly flat distributions of the cross sections in the three regions of $W \in[2.26,2.50],[2.50,2.62]$ and $[2.62,3.06] \mathrm{GeV}$ are consistent with the expectations from three-body final-state production via two-photon collisions. Both the peaking structures $[\gamma \gamma \rightarrow \eta(1760)$ or $X(1835) \rightarrow$ $\eta^{\prime} \pi^{+} \pi^{-}$and $\gamma \gamma \rightarrow \eta^{\prime} f_{0}(980) \rightarrow \eta^{\prime} \pi^{+} \pi^{-}$] follow a uniform angular distribution; thus, there is no distortion with or without their contribution in the resulting angular distribution in Fig. 14.

\section{Systematic uncertainty}

Systematic uncertainties arising from the pion identification, $\pi^{0}$-veto and $\eta^{\prime}$-sdb background in measurements of the cross sections for both $\gamma \gamma \rightarrow \eta^{\prime} \pi^{+} \pi^{-}$and $\gamma \gamma \rightarrow$ $\eta^{\prime} f_{2}(1270)$ production are estimated in each two-dimensional bin, using a method similar to that in the determination of the product of two-photon width and branching fraction for the final state, $\Gamma_{\gamma \gamma} \mathcal{B}$. The uncertainty in the trigger efficiency is calculated to be $1.2 \%$ $6.7 \%$ for the $\eta \pi \pi$ mode. The uncertainty in the determination of the $b_{\text {any }}$ background shape is estimated by changing each parameter by $\pm 1 \sigma$ in the fit, and the difference in yields with and without this change in each

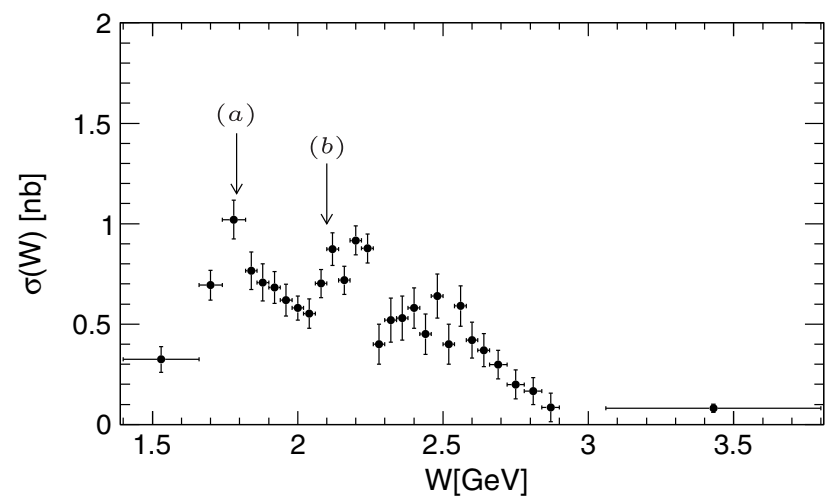

FIG. 13. Left panel: cross sections of $\gamma \gamma \rightarrow \eta^{\prime} \pi^{+} \pi^{-}$[including $\eta^{\prime} f_{2}(1270)$ ] (black solid dots) and $\gamma \gamma \rightarrow \eta^{\prime} f_{2}(1270)$ (red open dots). Right panel: cross sections of $\gamma \gamma \rightarrow \eta^{\prime} \pi^{+} \pi^{-}$[excluding $\gamma \gamma \rightarrow \eta^{\prime} f_{2}(1270)$ ] in the $W$ range above $2.26 \mathrm{GeV}$. The structure (a) near $1.8 \mathrm{GeV}$ arises from $X(1835)$ and $\eta(1760)$; the structure (b) near $2.1 \mathrm{GeV}$ is perhaps from $\gamma \gamma \rightarrow \eta^{\prime} f_{0}(980)$ production. In both panels, the error bars are statistical. 
TABLE V. Measured cross sections for $\gamma \gamma \rightarrow \eta^{\prime} \pi^{+} \pi^{-}$after subtracting contributions from $\gamma \gamma \rightarrow \eta^{\prime} f_{2}(1270)$ in the $W$ region above $2.26 \mathrm{GeV}$ and $\eta_{c}(1 S)$ in the $W$ region of $[2.62,3.06] \mathrm{GeV}$. The first error is statistical, and the second is systematic.

\begin{tabular}{lccc}
\hline \hline$W(\mathrm{GeV})$ & $\sigma\left(\gamma \gamma \rightarrow \eta^{\prime} \pi^{+} \pi^{-}\right)(\mathrm{nb})$ & $W(\mathrm{GeV})$ & $\sigma\left(\gamma \gamma \rightarrow \eta^{\prime} \pi^{+} \pi^{-}\right)(\mathrm{nb})$ \\
\hline $1.40-1.66$ & $0.315 \pm 0.064_{-0.046}^{+0.046}$ & $2.30-2.34$ & $0.52 \pm 0.11_{-0.10}^{+0.10}$ \\
$1.66-1.74$ & $0.689 \pm 0.074_{-0.088}^{+0.084}$ & $2.34-2.38$ & $0.53 \pm 0.11_{-0.10}^{+0.10}$ \\
$1.74-1.82$ & $1.01 \pm 0.10_{-0.11}^{+0.11}$ & $2.38-2.42$ & $0.58 \pm 0.10_{-0.11}^{+0.11}$ \\
$1.82-1.86$ & $0.77 \pm 0.09_{-0.11}^{+0.09}$ & $2.42-2.46$ & $0.45 \pm 0.10_{-0.09}^{+0.09}$ \\
$1.86-1.90$ & $0.69 \pm 0.09_{-0.10}^{+0.08}$ & $2.46-2.50$ & $0.64 \pm 0.11_{-0.14}^{+0.14}$ \\
$1.90-1.94$ & $0.661 \pm 0.082_{-0.091}^{+0.075}$ & $2.50-2.54$ & $0.40 \pm 0.10_{-0.08}^{+0.07}$ \\
$1.94-1.98$ & $0.62 \pm 0.08_{-0.12}^{+0.07}$ & $2.54-2.58$ & $0.59 \pm 0.10_{-0.11}^{+0.11}$ \\
$1.98-2.02$ & $0.58 \pm 0.060_{-0.082}^{+0.065}$ & $2.58-2.62$ & $0.42 \pm 0.09_{-0.09}^{+0.09}$ \\
$2.02-2.06$ & $0.552 \pm 0.072_{-0.094}^{+0.062}$ & $2.62-2.66$ & $0.37 \pm 0.08_{-0.07}^{+0.07}$ \\
$2.06-2.10$ & $0.70 \pm 0.07_{-0.17}^{+0.08}$ & $2.66-2.72$ & $0.30 \pm 0.07$ \\
$2.10-2.14$ & $0.85 \pm 0.08_{-0.09}^{+0.09}$ & $2.72-2.78$ & $0.20 \pm 0.07_{-0.04}^{+0.03}$ \\
$2.14-2.18$ & $0.71 \pm 0.07_{-0.12}^{+0.08}$ & $2.78-2.84$ & $0.17 \pm 0.07_{-0.03}^{+0.03}$ \\
$2.18-2.22$ & $0.92 \pm 0.07_{-0.11}^{+0.10}$ & $2.84-2.90$ & $0.085 \pm 0.071_{-0.015}^{+0.015}$ \\
$2.22-2.26$ & $0.86 \pm 0.07_{-0.11}^{+0.10}$ & $3.06-3.80$ & $0.081 \pm 0.021_{-0.022}^{+0.021}$ \\
$2.26-2.30$ & $0.40 \pm 0.10_{-0.08}^{+0.08}$ & & \\
\hline \hline
\end{tabular}

parameter, added in quadrature, is taken as its contribution to the systematic uncertainty. We study the non- $\eta^{\prime}$ events with the same final state of $\gamma \gamma \rightarrow \gamma \pi \pi \pi \pi$ in MC. We see that these non- $\eta^{\prime}$ events with a wrong combination of $\gamma \pi \pi$, surviving the $\eta^{\prime} \pi \pi$ selection criteria, have a peaking feature in the $\left|\Sigma p_{t}^{*}\right|$ distribution in the $\eta^{\prime}$ signal window. The contribution from non $-\eta^{\prime}$ is regarded as a lower systematic uncertainty of the cross section.
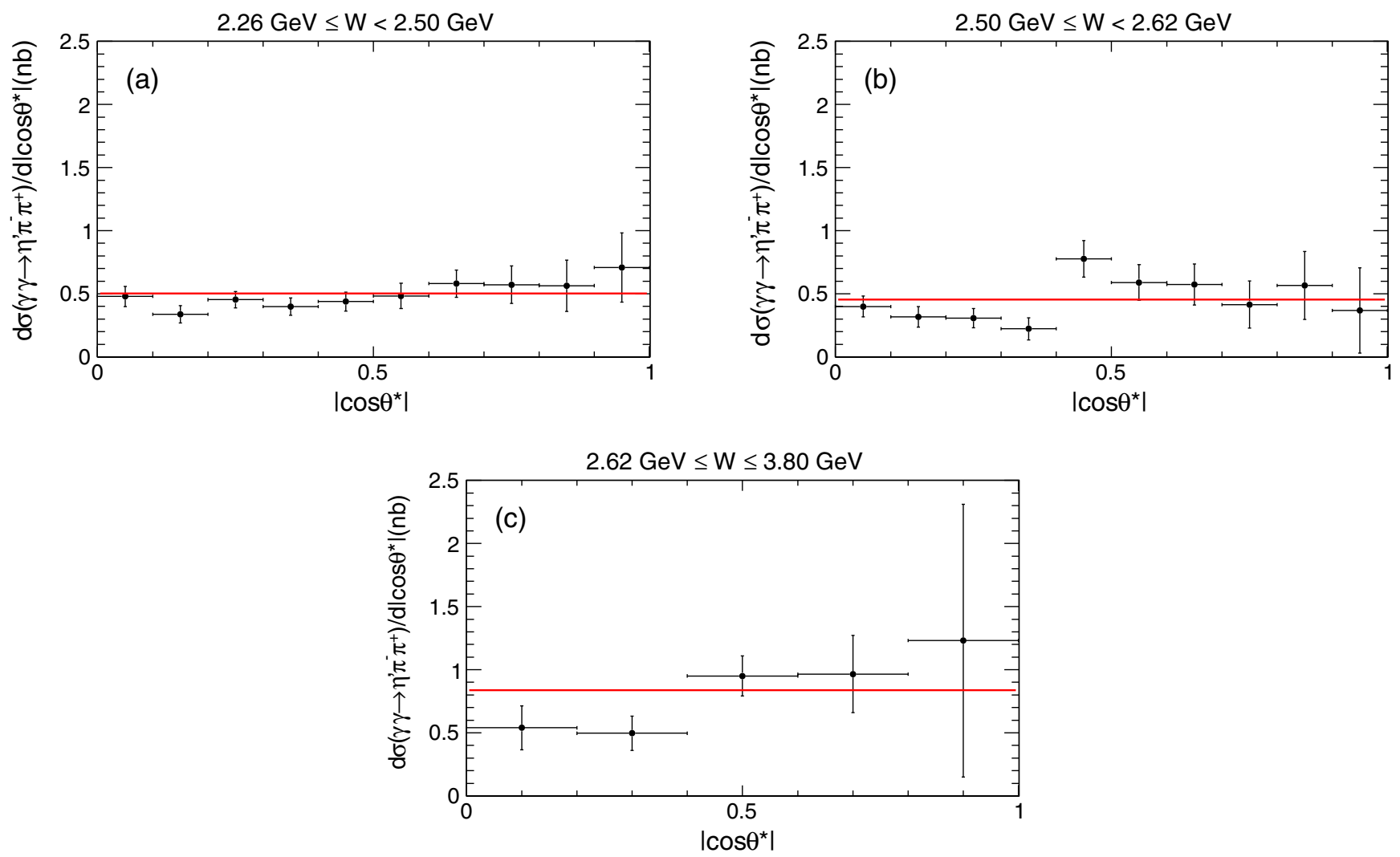

FIG. 14. Differential cross sections of $\gamma \gamma \rightarrow \eta^{\prime} \pi^{+} \pi^{-}$[excluding $\eta^{\prime} f_{2}(1270)$ ] in $\left|\cos \theta^{*}\right|$ in three $W$ regions from 2.26 to $3.80 \mathrm{GeV}$. The red solid line is a uniform distribution normalized to the data. In all panels, the error bars are statistical. 
TABLE VI. Summary of systematic uncertainties in the differential cross section measurement.

\begin{tabular}{lcc}
\hline \hline Source & $\eta^{\prime} \pi \pi(\%)$ & $\eta^{\prime} f_{2}(1270)(\%)$ \\
\hline Trigger efficiency & $1.2-6.7$ & $1.2-1.4$ \\
Background shape & $0.6-6.5$ & $12-21$ \\
$\eta^{\prime}-s d b$ and $b_{\text {any }}$ & $0.6-6.6$ & $1.6-2.1$ \\
$\pi^{0}$-veto & $2.7-4.4$ & $2.9-3.7$ \\
$\pi^{ \pm}$identification efficiency & $0.6-1.9$ & $0.8-1.8$ \\
non- $\eta^{\prime}$ & $2.0-21$ & $\ldots$ \\
$\eta$ reconstruction efficiency & \multicolumn{2}{c}{4.9} \\
Track reconstruction efficiency & \multicolumn{2}{c}{5.5} \\
Two-photon luminosity & \multicolumn{2}{c}{3} \\
Run dependence & \multicolumn{3}{c}{} \\
\hline \hline
\end{tabular}

The systematic uncertainties in the measurements of the cross sections are summarized in Table VI.

\section{SUMMARY AND DISCUSSIONS}

The $\eta_{c}(1 S), \eta_{c}(2 S)$, and nonresonant production of the $\eta^{\prime} \pi^{+} \pi^{-}$final state via two-photon collisions are measured. The results for the yields, masses, and widths, as well as the product decay widths are summarized in Table I for the $\eta_{c}(1 S)$ and $\eta_{c}(2 S)$. The differential cross sections for the nonresonant states of two-body $\eta^{\prime} f_{2}(1270)$ with $f_{2}(1270) \rightarrow \pi^{+} \pi^{-}$and three-body $\eta^{\prime} \pi^{+} \pi^{-}$[excluding $\left.\eta^{\prime} f_{2}(1270)\right]$ in the $\eta \pi \pi$ mode are shown in Tables IV and V and Figs. 11-14.

The $\eta_{c}(1 S)$ mass and width are measured to be $M=$ $[2984.6 \pm 0.7$ (stat) \pm 2.2 (syst) \pm 0.3 (model) $] \mathrm{MeV} / c^{2}$ and $\Gamma=\left[30.8_{-2.2}^{+2.3}\right.$ (stat) \pm 2.5 (syst) $\pm 1.4($ model $\left.)\right] \mathrm{MeV}$ and are consistent with the world-average values [33]. Here, the differences in the $\eta_{c}(1 S)$ mass and width with and without interference between $\eta_{c}(1 S)$ and nonresonant component, $\Delta M=0.3 \mathrm{MeV} / c^{2}$ and $\Delta \Gamma=1.4 \mathrm{MeV}$, are taken as model-dependent uncertainties in the determination of the mass and width [11]. The directly measured product of the two-photon width and branching fraction for $\eta_{c}(1 S)$ decay to $\eta^{\prime} \pi^{+} \pi^{-}$is determined to be $\Gamma_{\gamma \gamma} \mathcal{B}\left(\eta_{c}(1 S) \rightarrow \eta^{\prime} \pi^{+} \pi^{-}\right)=(65.4 \pm 2.6 \pm 7.8) \mathrm{eV}$. By employing the full $\Upsilon(4 S)$ and $\Upsilon(5 S)$ data samples $\left(941 \mathrm{fb}^{-1}\right)$ and an additional decay mode for the $\eta^{\prime} \rightarrow \gamma \rho$, the results for the $\eta_{c}(1 S)$ mass, width and product of its decay width in this measurement are obtained with improved statistical errors, and thus supersede our previous measurement using a $673 \mathrm{fb}^{-1}$ data sample [11]. With the world-average value of $\Gamma_{\gamma \gamma}\left(\eta_{c}(1 S)\right)=(5.1 \pm 0.4) \mathrm{keV}$ [33] as input, the branching fraction is calculated to be $\mathcal{B}\left(\eta_{c}(1 S) \rightarrow \eta^{\prime} \pi^{+} \pi^{-}\right)=$

$[12.8 \pm 0.5$ (stat) \pm 1.4 (syst) $\pm 1.0(\mathrm{PDG})] \times 10^{-3}$, where the third error is due to the $\eta_{c}(1 S)$ two-photon decay width.

We report the first observation of $\eta_{c}(2 S) \rightarrow \eta^{\prime} \pi^{+} \pi^{-}$, with a significance of $5.5 \sigma$ including the systematic error. We measure the mass of the $\eta_{c}(2 S)$ to be $M=$ $[3635.1 \pm 3.7$ (stat) \pm 2.9 (syst) \pm 0.4 (model) $] \mathrm{MeV} / \mathrm{c}^{2}$, which is consistent with the world-average value [33], and the product of two-photon width and branching fraction to $\eta^{\prime} \pi^{+} \pi^{-}$to be $\Gamma_{\gamma \gamma} \mathcal{B}\left(\eta_{c}(2 S) \rightarrow \eta^{\prime} \pi^{+} \pi^{-}\right)=$ $\left(5.6_{-1.1}^{+1.2} \pm 1.1\right) \mathrm{eV}$.

In fact, the ratio of the two products of two-photon decay width and branching fraction for the $\eta_{c}(1 S)$ and $\eta_{c}(2 S)$,

$$
\mathcal{R}=\frac{\Gamma_{\gamma \gamma}\left(\eta_{c}(2 S)\right) \mathcal{B}\left(\eta_{c}(2 S)\right)}{\Gamma_{\gamma \gamma}\left(\eta_{c}(1 S)\right) \mathcal{B}\left(\eta_{c}(1 S)\right)}
$$

is a quantity directly measured in experiments. The $\eta_{c}(1 S)$ and $\eta_{c}(2 S)$ mesons in the measurements are all produced via two-photon process, and the dominant contributions to the systematic uncertainty in either product alone, such as those for the two-photon luminosity and reconstruction efficiencies of $\eta$ and charged pion tracks, cancel almost completely in this ratio. As shown in Table VII, the $\mathcal{R}$ values from the two observations-one by BABAR [6] with $K \bar{K} \pi$ and the other by this analysis with $\eta^{\prime} \pi^{+} \pi^{-}-$ are measured to be $\mathcal{R}=(10.6 \pm 2.0) \times 10^{-2}$ and $(8.6 \pm 2.7) \times 10^{-2}$, respectively. They are consistent with each other, while a third measurement with large uncertainty by CLEO [9] is compatible with the former. It implies that the assumption of approximate equality of the branching fractions for $\eta_{c}(1 S)$ and $\eta_{c}(2 S)$ to a specific final state,

TABLE VII. Comparison of the $\Gamma_{\gamma \gamma} \mathcal{B}$ for $\eta_{c}(1 S)$ and $\eta_{c}(2 S)$ decays by CLEO, Belle, and $B A B A R$, along with the ratio $\mathcal{R}\left(\eta_{c}(2 S) / \eta_{c}(1 S)\right)=\left(\Gamma_{\gamma \gamma}\left(\eta_{c}(2 S)\right) \mathcal{B}\left(\eta_{c}(2 S)\right)\right) /\left(\Gamma_{\gamma \gamma}\left(\eta_{c}(1 S)\right) \mathcal{B}\left(\eta_{c}(1 S)\right)\right)$. The two-photon decay width $\Gamma_{\gamma \gamma}\left(\eta_{c}(2 S)\right.$ is estimated using the world-average value of $\Gamma_{\gamma \gamma}\left(\eta_{c}(1 S)\right)=(5.1 \pm 0.4) \mathrm{keV}$ as input under the assumption of equal $\mathcal{B}$ for $\eta_{c}(1 S)$ and $\eta_{c}(2 S)$ decays.

\begin{tabular}{lccccc}
\hline \hline Final state & $\Gamma_{\gamma \gamma} \mathcal{B}$ for $\eta_{c}(1 S)$ & $\Gamma_{\gamma \gamma} \mathcal{B}$ for $\eta_{c}(2 S)$ & $\mathcal{R}\left(\eta_{c}(2 S) / \eta_{c}(1 S)\right)$ & $\Gamma_{\gamma \gamma}\left(\eta_{c}(2 S)\right)$ & $(\mathrm{keV})$ \\
\hline$K_{S}^{0} K^{+} \pi^{-}$ & $(\mathrm{eV})$ & $(\mathrm{eV})$ & $\left(\times 10^{-2}\right)$ & Reference \\
$K \bar{K} \pi$ & $\ldots$ & $\ldots$ & $18 \pm 5 \pm 2$ & $0.92 \pm 0.28$ & {$[9] \mathrm{CLEO} 2004$} \\
$\eta^{\prime} \pi^{+} \pi^{-}$ & $386 \pm 8 \pm 21$ & $41 \pm 4 \pm 6$ & $10.6 \pm 2.0$ & $0.54 \pm 0.11$ & {$[6] B A B A R 2011$} \\
$\mathrm{QCD}$ & $65.4 \pm 2.6 \pm 7.8$ & $5.6 \pm 1.2 \pm 1.1$ & $8.6 \pm 2.7$ & $0.44 \pm 0.14$ & This, Belle \\
& & & & $1.8-5.7$ & {$[12-17] 1992-2005$} \\
\hline \hline
\end{tabular}




$$
\frac{\mathcal{B}\left(\eta_{c}(2 S) \rightarrow \eta^{\prime} \pi^{+} \pi^{-}\right)}{\mathcal{B}\left(\eta_{c}(1 S) \rightarrow \eta^{\prime} \pi^{+} \pi^{-}\right)} \cong \frac{\mathcal{B}\left(\eta_{c}(2 S) \rightarrow K \bar{K} \pi\right)}{\mathcal{B}\left(\eta_{c}(1 S) \rightarrow K \bar{K} \pi\right)}
$$

is reasonable within the errors. Here, the systematic uncertainty contributions in the $\mathcal{R}$ values [and thus the ratio of branching fractions for $\eta_{c}(1 S)$ and $\eta_{c}(2 S)$ decays in Eq. (9)] are conservatively estimated, since their cancellation effect in determination of the ratio $\mathcal{R}$ errors is not subtracted yet.

Under the assumption of equal branching fractions for $\eta_{c}(1 S)$ and $\eta_{c}(2 S)$ decay, the two-photon decay width for $\eta_{c}(2 S)$ is determined to be $\Gamma_{\gamma \gamma}\left(\eta_{c}(2 S)\right)=(1.3 \pm 0.6) \mathrm{keV}$ by CLEO [9], which lies at the lower bound of the QCD predictions [12-17]. The resulting $\Gamma_{\gamma \gamma}\left(\eta_{c}(2 S)\right)$ value, derived from this work, is less than half of CLEO's (see Table VII). On the other hand, the measured unequal branching fractions for $\eta_{c}(1 S)$ and $\eta_{c}(2 S)$ decays to $K \bar{K} \pi$, albeit with good precision for the former [33] but large uncertainty for the latter [10], indicates that an improved test of the assumption with experimental data is indeed needed. Precision measurements of the branching fraction for either $\eta_{c}(2 S)$ decays to $K_{S}^{0} K^{+} \pi^{-}\left(\eta \pi^{+} \pi^{-}\right)$or $B$ decays to $K \eta_{c}(2 S)$ would be able to clarify the discrepancy in the two-photon decay width of $\eta_{c}(2 S)$ between data and QCD predictions.

The cross sections of $\gamma \gamma \rightarrow \eta^{\prime} f_{2}(1270)$ and $\gamma \gamma \rightarrow \eta^{\prime} \pi^{+} \pi^{-}$ [excluding $\left.\eta^{\prime} f_{2}(1270)\right]$ in $\eta \pi^{+} \pi^{-}$mode are measured. Under the assumption of the power law dependence $\sigma \propto$ $1 /\left(W^{n} \cdot \sin ^{\alpha} \theta^{*}\right)$ for pseudoscalar tensor meson pair production, the fitted index $n=7.5 \pm 2.0$ (for $\left|\cos \theta^{*}\right|<0.6$ ) shows that the cross section of the $\gamma \gamma \rightarrow \eta^{\prime} f_{2}(1270)$ production with $\eta^{\prime}$ scattering at large angles in the $\gamma \gamma$ rest system behaves much steeper in its $W$ dependence than that at small angle, and that the $W$ dependence of cross section in the power law is compatible, within error, with the sharply dropping behavior for neutral pseudoscalar meson pair production measured by Belle $(n=7.8-11)$ [22] and predicted by QCD $(n=10)$ [18-21]. On the other hand, the behavior of the cross sections' angular dependence for the ranges of $W \in[2.50,2.62]$ and $\in[2.62,3.8] \mathrm{GeV}$ is compatible with that for $\pi^{0} \pi^{0}$ and $\eta \pi^{0}$ production as measured by Belle [22] and with that for pseudoscalar meson pair production predicted by the QCD calculations [18-21].

In summary, the $\eta_{c}(1 S), \eta_{c}(2 S)$ and nonresonant $\eta^{\prime} \pi^{+} \pi^{-}$ production via two-photon collisions is measured. We report the first observation of the signal for $\eta_{c}(2 S)$ decays to $\eta^{\prime} \pi^{+} \pi^{-}$, the measured products of the two-photon decay width and the branching fraction for the $\eta_{c}(1 S)$ and $\eta_{c}(2 S)$ decays to $\eta^{\prime} \pi^{+} \pi^{-}$, and the measurement of nonresonant production of two-body $\eta^{\prime} f_{2}(1270)$ and three-body $\eta^{\prime} \pi^{+} \pi^{-}$ final states via two-photon collisions.

\section{ACKNOWLEDGMENTS}

We extend our special thanks to Y. H. Zheng and X. R. Lyu of the University of Chinese Academy of Sciences for helpful discussions. We thank the KEKB group for the excellent operation of the accelerator; the KEK cryogenics group for the efficient operation of the solenoid; and the KEK computer group, the National Institute of Informatics, and the Pacific Northwest National Laboratory (PNNL) Environmental Molecular Sciences Laboratory (EMSL) computing group for valuable computing and Science Information NETwork 5 (SINET5) network support. We acknowledge support from the Ministry of Education, Culture, Sports, Science, and Technology (MEXT) of Japan, the Japan Society for the Promotion of Science (JSPS), and the Tau-Lepton Physics Research Center of Nagoya University; the Australian Research Council; Austrian Science Fund under Grant No. P 26794-N20; the National Natural Science Foundation of China under Contracts No. 11435013, No. 11475187, No. 11521505, No. 11575017, No. 11675166, No. 11705209; Key Research Program of Frontier Sciences, Chinese Academy of Sciences (CAS), Grant No. QYZDJ-SSW-SLH011; the CAS Center for Excellence in Particle Physics (CCEPP); Fudan University Grants No. JIH5913023, No. IDH5913011/003, No. JIH5913024, No. IDH5913011/002; the Ministry of Education, Youth and Sports of the Czech Republic under Contract No. LTT17020; the Carl Zeiss Foundation, the Deutsche Forschungsgemeinschaft, the Excellence Cluster Universe, and the VolkswagenStiftung; the Department of Science and Technology of India; the Istituto Nazionale di Fisica Nucleare of Italy; National Research Foundation (NRF) of Korea Grants No. 2014R1A2A2A01005286, No. 2015R1A2A2A01003280, No. 2015H1A2A1033649, No. 2016R1D1A1B01010135, No. 2016K1A3A7A09005 603, No. 2016R1D1A1B02012900; Radiation Science Research Institute, Foreign Large-size Research Facility Application Supporting project and the Global Science Experimental Data Hub Center of the Korea Institute of Science and Technology Information; the Polish Ministry of Science and Higher Education and the National Science Center; the Ministry of Higher Education and Science of the Russian Federation under the grant 14.W03.31.0026; the Slovenian Research Agency; Ikerbasque, Basque Foundation for Science, Basque Government (No. IT95616) and Ministry of Economy and Competitiveness (MINECO) (Juan de la Cierva), Spain; the Swiss National Science Foundation; the Ministry of Education and the Ministry of Science and Technology of Taiwan; and the United States Department of Energy and the National Science Foundation. 
[1] N. Brambilla et al., Eur. Phys. C 71, 1534 (2011).

[2] J. P. Lansberg and T. N. Pham, Phys. Rev. D 74, 034001 (2006).

[3] N. Brambilla, A. Pineda, J. Soto, and A. Vairo, Rev. Mod. Phys. 77, 1423 (2005).

[4] M. Ablikim et al. (BESIII Collaboration), Phys. Rev. Lett. 108, 222002 (2012).

[5] M. Ablikim et al. (BESIII Collaboration), Phys. Rev. D 86, 092009 (2012); Phys. Rev. Lett. 109, 042003 (2012).

[6] P. del Amo Sanchez et al. (BABAR Collaboration), Phys. Rev. D 84, 012004 (2011).

[7] A. Vinokurova et al. (Belle Collaboration), Phys. Lett. B 706, 139 (2011).

[8] S. Uehara et al. (Belle Collaboration), Eur. Phys. J. C 53, 1 (2008).

[9] D. M. Asner et al. (CLEO Collaboration), Phys. Rev. Lett. 92, 142001 (2004). The value $1.3 \pm 0.6 \mathrm{keV}$ by CLEO is calculated using $\Gamma_{\gamma \gamma}\left(\eta_{c}(1 S)\right)=(7.4 \pm 0.4 \pm 2.3) \mathrm{keV}$ of the CLEO's as input.

[10] B. Aubert et al. (BABAR Collaboration), Phys. Rev. D 78, 012006 (2008).

[11] C. C. Zhang et al. (Belle Collaboration), Phys. Rev. D 86, 052002 (2012).

[12] E. S. Ackleh and T. Barnes, Phys. Rev. D 45, 232 (1992).

[13] M. R. Ahmady and R. R. Mendel, Phys. Rev. D 51, 141 (1995).

[14] C. R. Munz, Nucl. Phys. A609, 364 (1996).

[15] H. W. Huang, J. H. Liu, J. Tang, and K. T. Chao, Phys. Rev. D 56, 368 (1997).

[16] D. Ebert, R. N. Faustov, and V. O. Galkin, Mod. Phys. Lett. A 18, 601 (2003).

[17] C. S. Kim, T. Lee, and G. L. Wang, Phys. Lett. B 606, 323 (2005).

[18] S. J. Brodsky and G. P. Lepage, Phys. Rev. D 24, 1808 (1981).

[19] V. L. Chernyak and A. R. Zhitnitsky, Phys. Rep. 112, 173 (1984).
[20] M. Benayoun and V. L. Chernyak, Nucl. Phys. B329, 285 (1990).

[21] M. Diehl, P. Kroll, and C. Vogt, Phys. Lett. B 532, 99 (2002).

[22] A. J. Bevan, B. Golob, Th. Mannel, S. Prell, B. D. Yabsley et al., Eur. Phys. Jour. C 74, 3026 (2014); see Sec. 22. 2. 2.

[23] A. Abashian et al. (Belle Collaboration), Nucl. Instrum. Methods Phys. Res., Sect. A 479, 117 (2002); also see detector section in J.Brodzicka et al., Prog. Theor. Exp. Phys. 2012, 04D001 (2012); S. Kurokawa and E. Kikutani, Nucl. Instrum. Methods Phys. Res., Sect. A 499, 1 (2003) and other papers included in this volume; T. Abe et al., Prog. Theor. Exp. Phys. 2013, 03A001 (2013) and references therein.

[24] S. Uehara, KEK Report 96-11 (1996).

[25] T. Sjöstrand, Comput. Phys. Commun. 82, 74 (1994).

[26] R. Brun et al., CERN Report No. DD/EE/84-1, 1984.

[27] T. Barnes, T. E. Browder, and S. F. Tuan, Phys. Lett. B 385, 391 (1996).

[28] V. M. Budnev, I. F. Ginzburg, G. V. Meledin, and V. G. Serbo, Phys. Rep. C 15, 181 (1975).

[29] J. Field, Nucl. Phys. B168, 477 (1980); and B176, 545 (1980).

[30] The original Crystal Ball (CB) function, defined in MINUIT, has a Gaussian in its central and upper-side regions and a tail in the lower side. The improved Crystal Ball (ICB) function is defined as a CB with an additional tail in its upper side.

[31] S. Uehara et al. (Belle Collaboration), Prog. Theor. Exp. Phys. 2013, 123C01 (2013).

[32] M. Masuda et al. (Belle Collaboration), Phys. Rev. D 93, 032003 (2016).

[33] C. Patrignani et al. (Particle Data Group), Chin. Phys. C 40, 100001 (2016).

[34] J. P. Lansberg and T. N. Pham, AIP Conf. Proc. 1038, 259 (2008). 